\title{
Thoracic and cardiovascular surgeries in Japan during 2017
}

\author{
Annual report by the Japanese Association for Thoracic Surgery
}

\begin{abstract}
Committee for Scientific Affairs, The Japanese Association for Thoracic Surgery ${ }^{1}$ - Hideyuki Shimizu ${ }^{2}$. Morihito Okada ${ }^{3}$ - Akira Tangoku ${ }^{4}$ - Yuichiro Doki ${ }^{5}$ - Shunsuke Endo ${ }^{6}$ - Hirotsugu Fukuda ${ }^{7}$.

Yasutaka Hirata $^{8}$ - Hisashi Iwata ${ }^{9}$. Junjiro Kobayashi ${ }^{10}$. Hiraku Kumamaru ${ }^{11}$. Hiroaki Miyata ${ }^{12}$. Noboru Motomura $^{13}$. Shoji Natsugoe ${ }^{14}$. Soji Ozawa ${ }^{15}$ - Yoshikatsu Saiki ${ }^{16}$ - Aya Saito $^{13}$. Hisashi Saji ${ }^{17}$. Yukio Sato $^{18} \cdot$ Tsuyoshi Taketani $^{19} \cdot$ Kazuo Tanemoto $^{20} \cdot$ Wataru Tatsuishi $^{21}$ - Yasushi Toh ${ }^{22}$. Hiroyuki Tsukihara $^{8} \cdot$ Masayuki Watanabe $^{23} \cdot$ Hiroyuki Yamamoto $^{12} \cdot$ Kohei Yokoi $^{24} \cdot$ Yutaka Okita $^{25}$
\end{abstract}

Published online: 5 March 2020

(C) The Author(s) 2020

The Japanese Association for Thoracic Surgery has conducted annual surveys of thoracic surgery throughout Japan since 1986 to determine statistics pertaining to the number of procedures performed, based on surgical category. Herein, we summarize the results of the association's annual survey of thoracic surgery performed in 2017.

Adhering to the norm to date, thoracic surgery was classified into three categories: cardiovascular, general thoracic, and esophageal surgeries. Patient data were examined and analyzed for each group. Access to computerized data is available to all members of the association. We honor and value all members' continued professional support and contributions (Tables 1, 2).

Incidence of hospital mortality was included in the survey to determine nationwide status, which has contributed to Japanese surgeons' understanding of the present status of thoracic surgery in Japan, while helping to effect progress for improving operative results by enabling them to compare their work with that of others. In this way, the association has been able to gain a better understanding of present problems and future prospects, which is reflected in its activities and the education of its members.

Thirty-day mortality (so-called operative mortality) is defined as death within 30 days of surgery, regardless of the patient's geographic location, including after the patient is discharged from hospital. Hospital mortality is

Morihito Okada and Akira Tangoku have equally contributed to this work.

Hideyuki Shimizu

survey-adm@umin.net

Extended author information available on the last page of the article defined as death within any time interval following surgery if the patient has not been discharged from hospital.

Hospital-to-hospital transfer in the category of esophageal surgery is not considered a form of discharge; transfer to a nursing home or a rehabilitation unit is considered hospital discharge, unless the patient subsequently dies because of complications from surgery. Contrastingly, hospital-to-hospital transfer 30 days following surgery in the categories of cardiovascular and general thoracic surgery is considered discharge, as data related to the National Clinical Database (NCD 2017) were employed in this category, and hospital-to-hospital transfer 30 days following surgery is considered discharge according to the NCD.

\section{Survey abstract}

All data pertaining to cardiovascular and thoracic surgeries were obtained from the NCD, whereas data regarding esophageal surgery were collected from a survey questionnaire derived from the Japanese Association for Thoracic Surgery documentation. The reason for this was that NCD information regarding esophageal surgery does not include non-surgical cases (i.e., patients with adjuvant chemotherapy or radiation only).

Because of changes in data collection related to cardiovascular surgery (initially self-reported using questionnaire sheets in each participating institution up to 2014, then by downloading an automatic package from the Japanese Cardiovascular Surgery Database (JCVSD), a cardiovascular sub-section of the NCD), the response rate is not available and is, therefore, not indicated in the cardiovascular surgery category (Table 1). Additionally, the number of institutions (based on surgery count) was not calculated in the cardiovascular surgery category (Table 2). 
Table 1 Number of institutions involved in the survey

\begin{tabular}{llll}
\hline & \multicolumn{2}{l}{ Questionnaires } \\
\cline { 2 - 4 } & Sent out & Responded & Response rate (\%) \\
\hline (A) Cardiovascular surgery & - & - & - \\
(B) General thoracic surgery & 740 & 678 & 91.6 \\
(C) Esophageal surgery & 568 & 523 & 92.1 \\
\hline
\end{tabular}

Table 2 Categories subclassified according to the number of operations performed

\begin{tabular}{lc}
\hline Number of operations performed & $\begin{array}{l}\text { Category } \\
\text { General thoracic surgery }\end{array}$ \\
\hline 0 & 6 \\
$1-24$ & 28 \\
$25-49$ & 72 \\
$50-99$ & 167 \\
$100-149$ & 129 \\
$150-199$ & 100 \\
$\geq 200$ & 176 \\
Total & 678 \\
\hline Number of operations performed & \\
\hline 0 & Esophageal surgery \\
$1-4$ & 76 \\
$5-9$ & 114 \\
$10-19$ & 96 \\
$20-29$ & 98 \\
$30-39$ & 47 \\
$40-49$ & 26 \\
$\geq 50$ & 21 \\
Total & 45 \\
\hline & 523 \\
\hline
\end{tabular}

\section{Final report: 2017}

\section{(A) Cardiovascular surgery}

We are extremely pleased with the cooperation of our colleagues (members) in terms of completing the cardiovascular surgery survey, thereby undoubtedly improving the quality of this annual report. We are truly grateful for the significant efforts made by all within each participating institution in completing the JCVSD/NCD.

Figure 1 illustrates the development of cardiovascular surgery in Japan over the past 30 years. Aneurysm surgery includes only surgeries for thoracic and thoracoabdominal aortic aneurysms. Extra-anatomic bypass surgery for thoracic aneurysm and pacemaker implantation has been excluded from the survey since 2015. The number of assist device implantation surgeries is not included in the total number of surgical procedures but was nonetheless included in the survey.

A total of 70,078 cardiovascular surgeries including 56 heart transplants were performed in 2017, an increase of $3.3 \%$ compared with that in the 2016 survey results $(n=$ $67,867)$. The number of cardiovascular surgeries is continuously increasing, despite an apparent decrease in 2015, likely due to major changes in data collection and aggregation approaches.

When compared with data for 2016 [1] and 2007 [2], the number of surgeries in 2017 for congenital heart disease increased by $7.1 \%$ (9368 vs. 8744 ) and $0.2 \%$, respectively; procedures for valvular heart disease increased by $0.2 \%$ $(23,312$ vs. 23,254$)$ and $53.2 \%$, respectively; surgery for thoracic aortic aneurysm increased by $8.7 \%$ (20,746 vs. 19,078 ) and $114.6 \%$, respectively; ischemic heart procedures decreased by $6.6 \%(13,898$ vs. 14,874$)$ and $23.6 \%$,

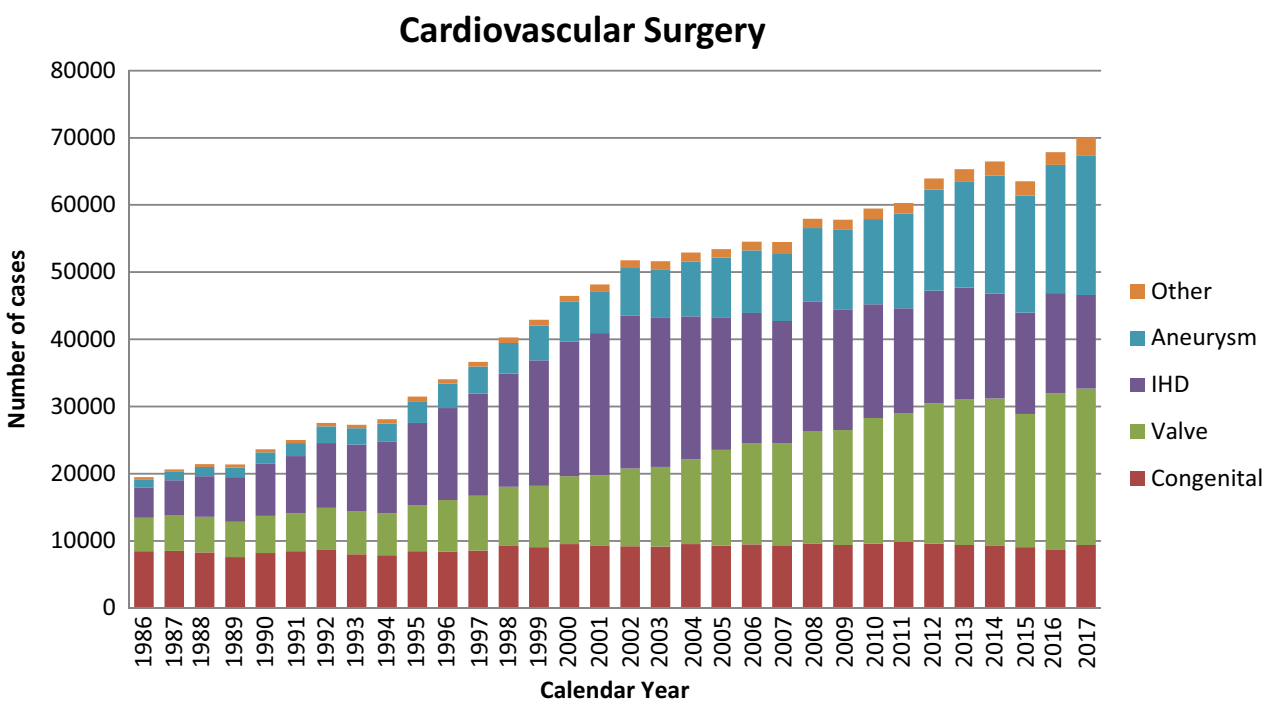

Fig. 1 Cardiovascular surgery. $I H D$ ischemic heart disease 


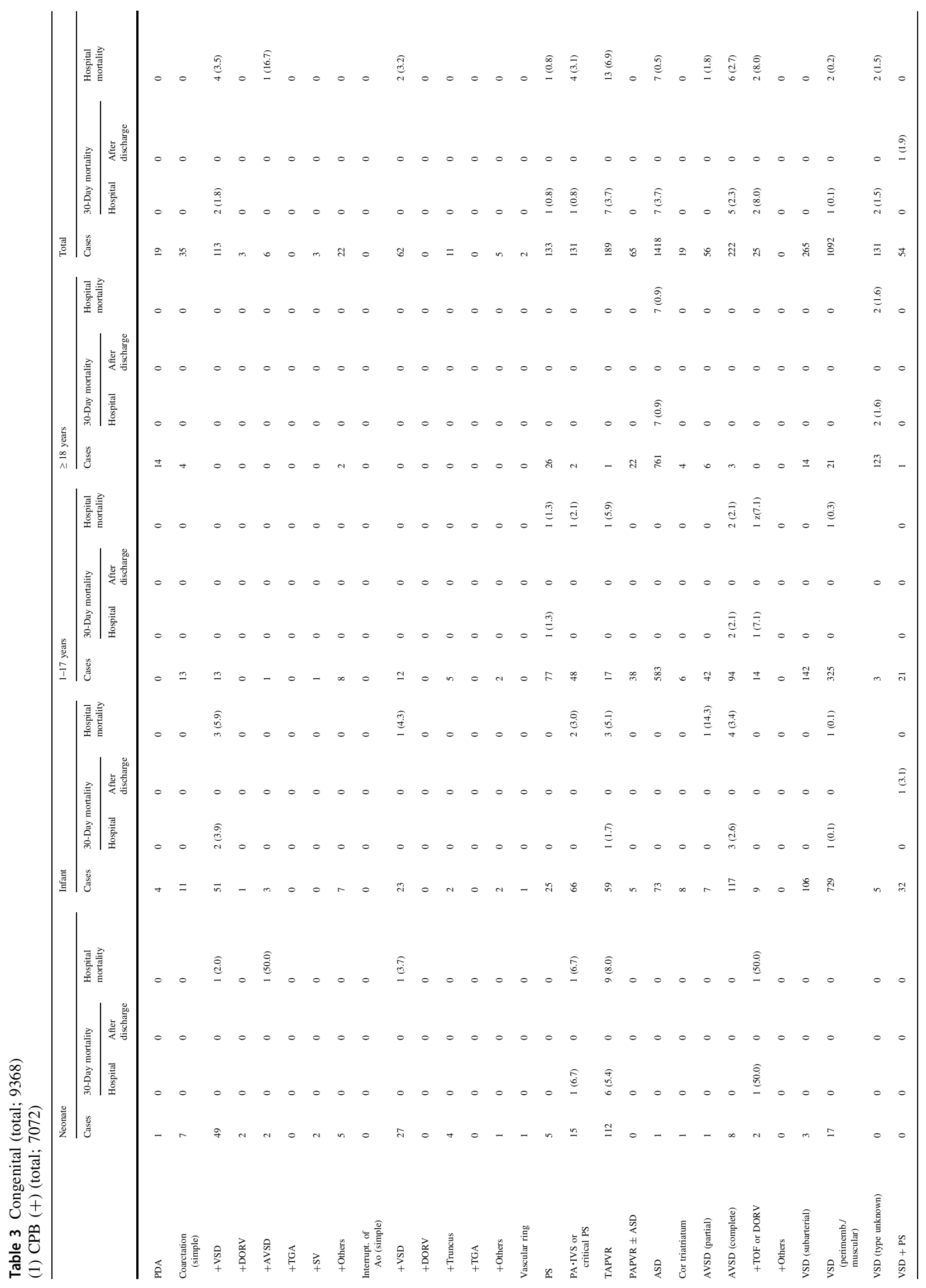




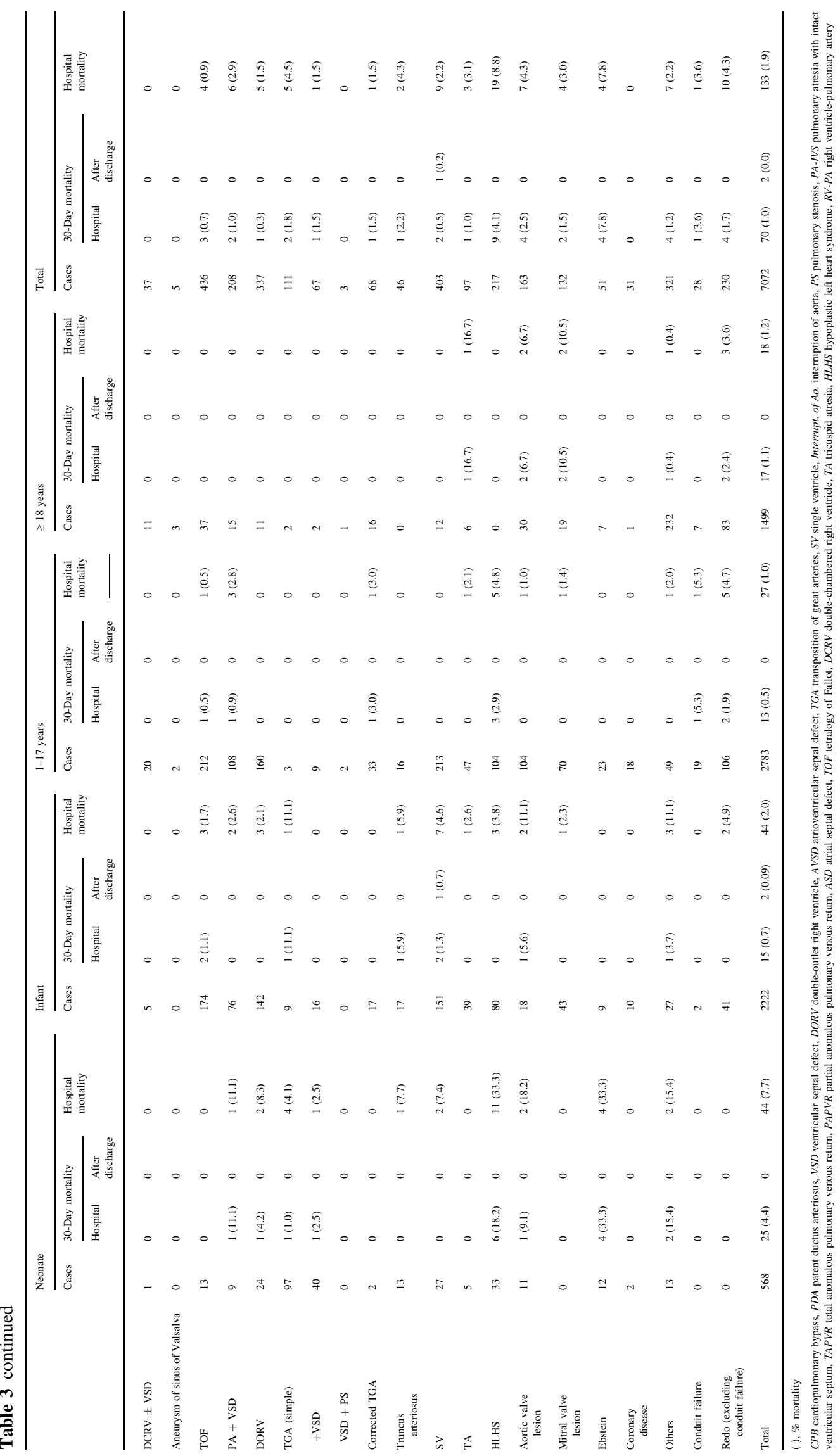




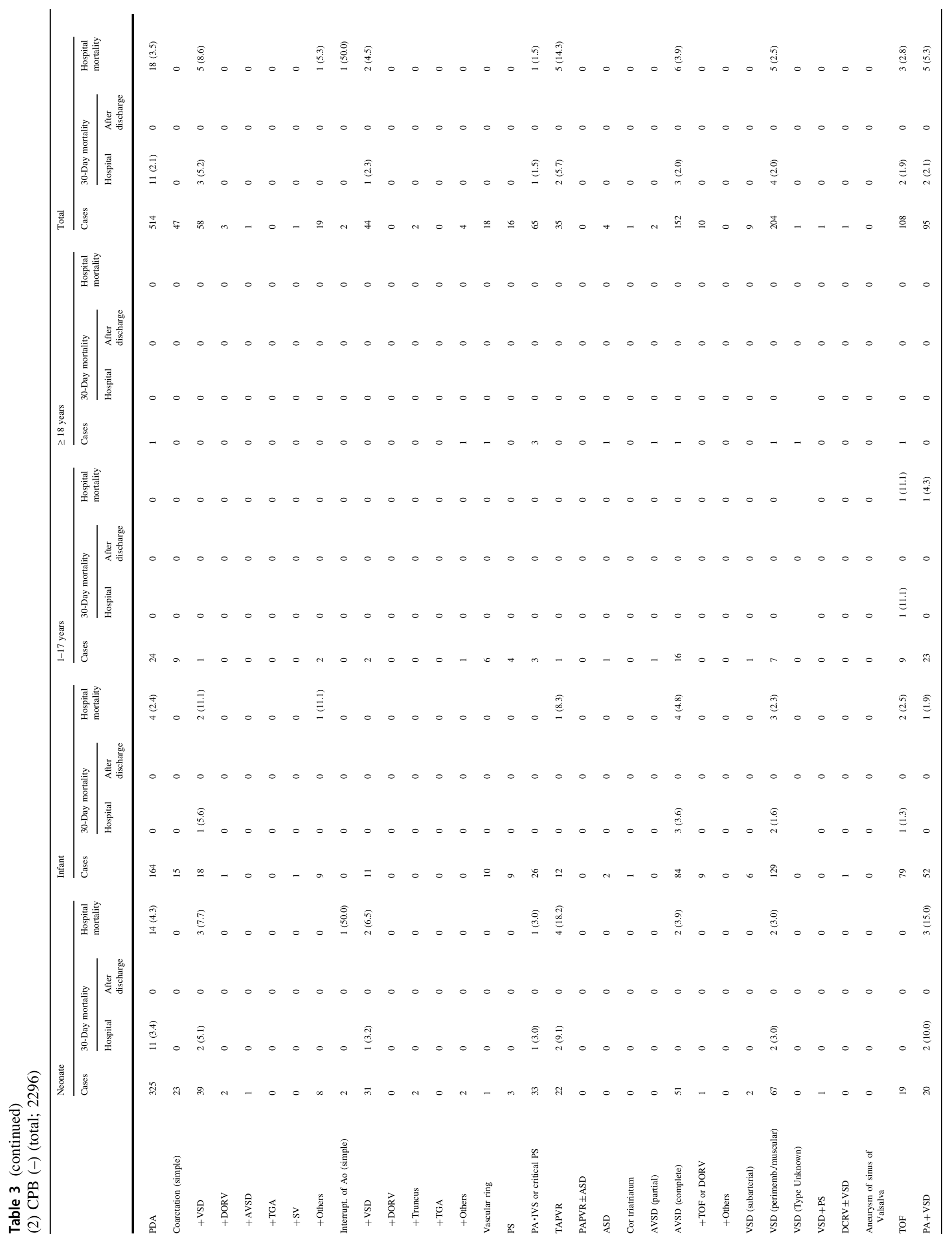


General Thoracic and Cardiovascular Surgery (2020) 68:414-449

419

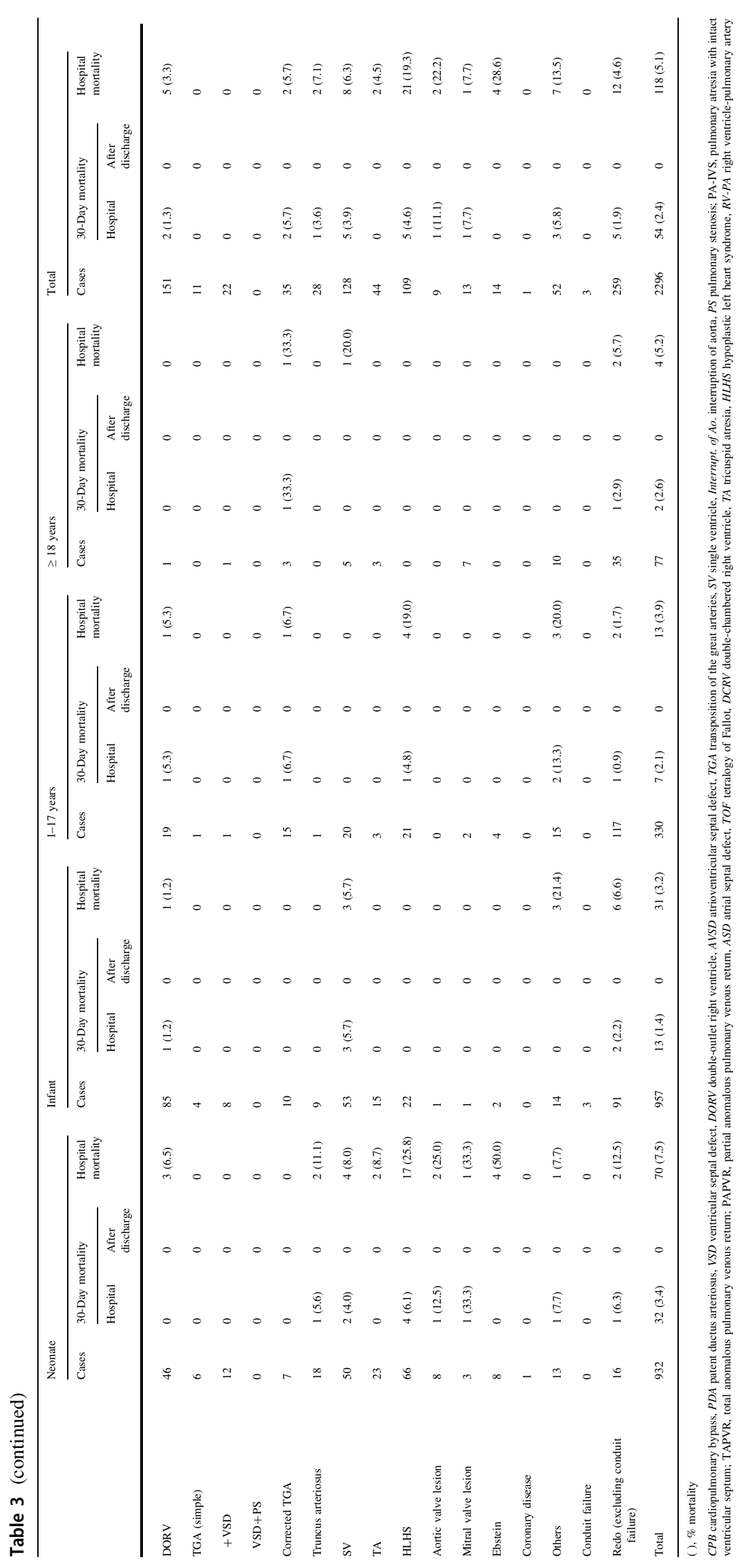

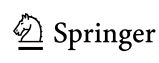




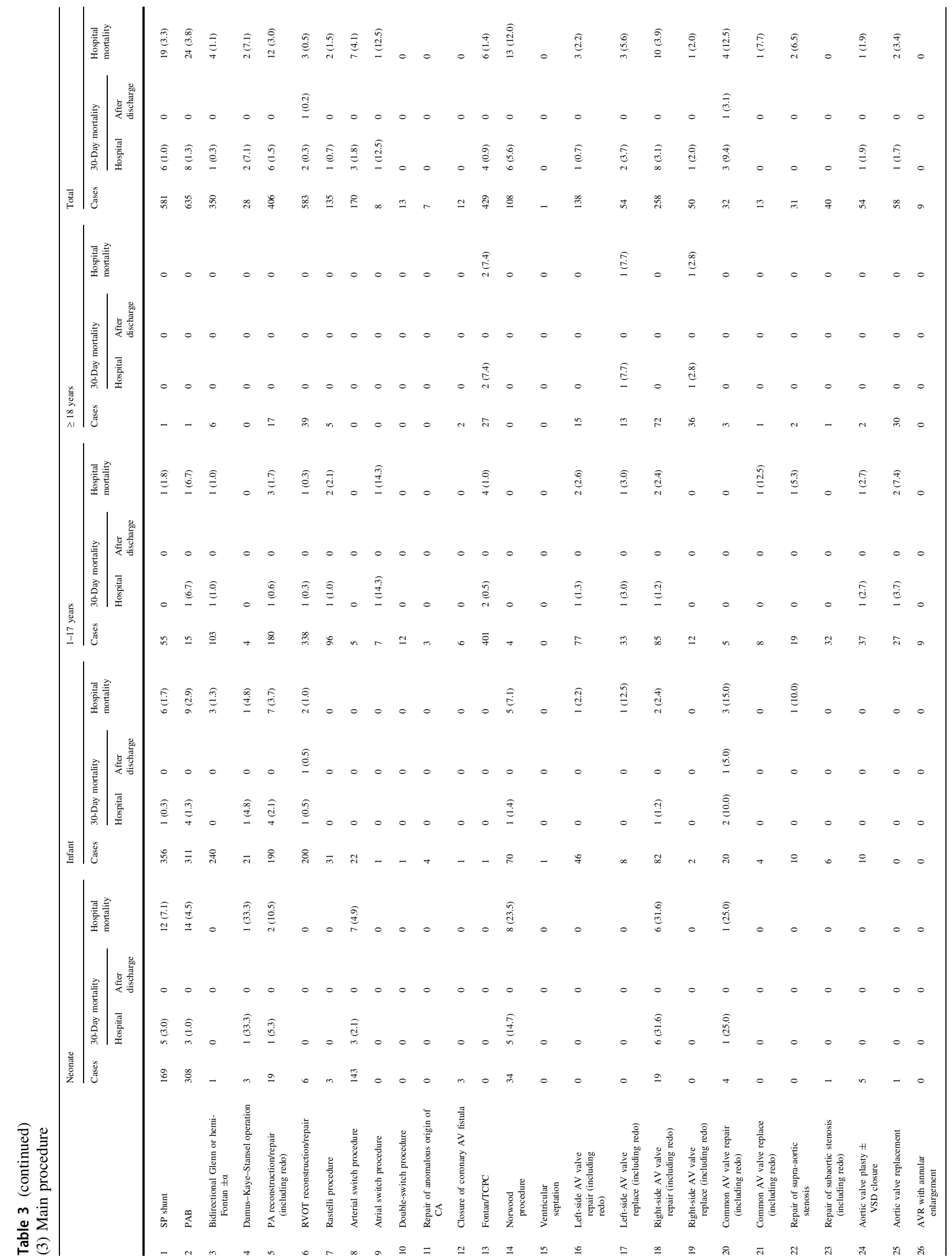


General Thoracic and Cardiovascular Surgery (2020) 68:414-449

421

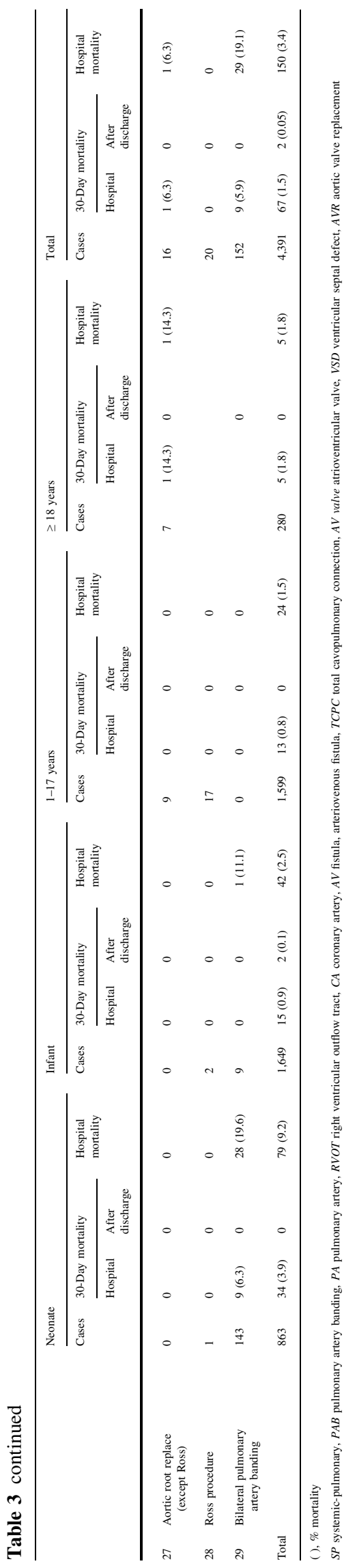

Springer 


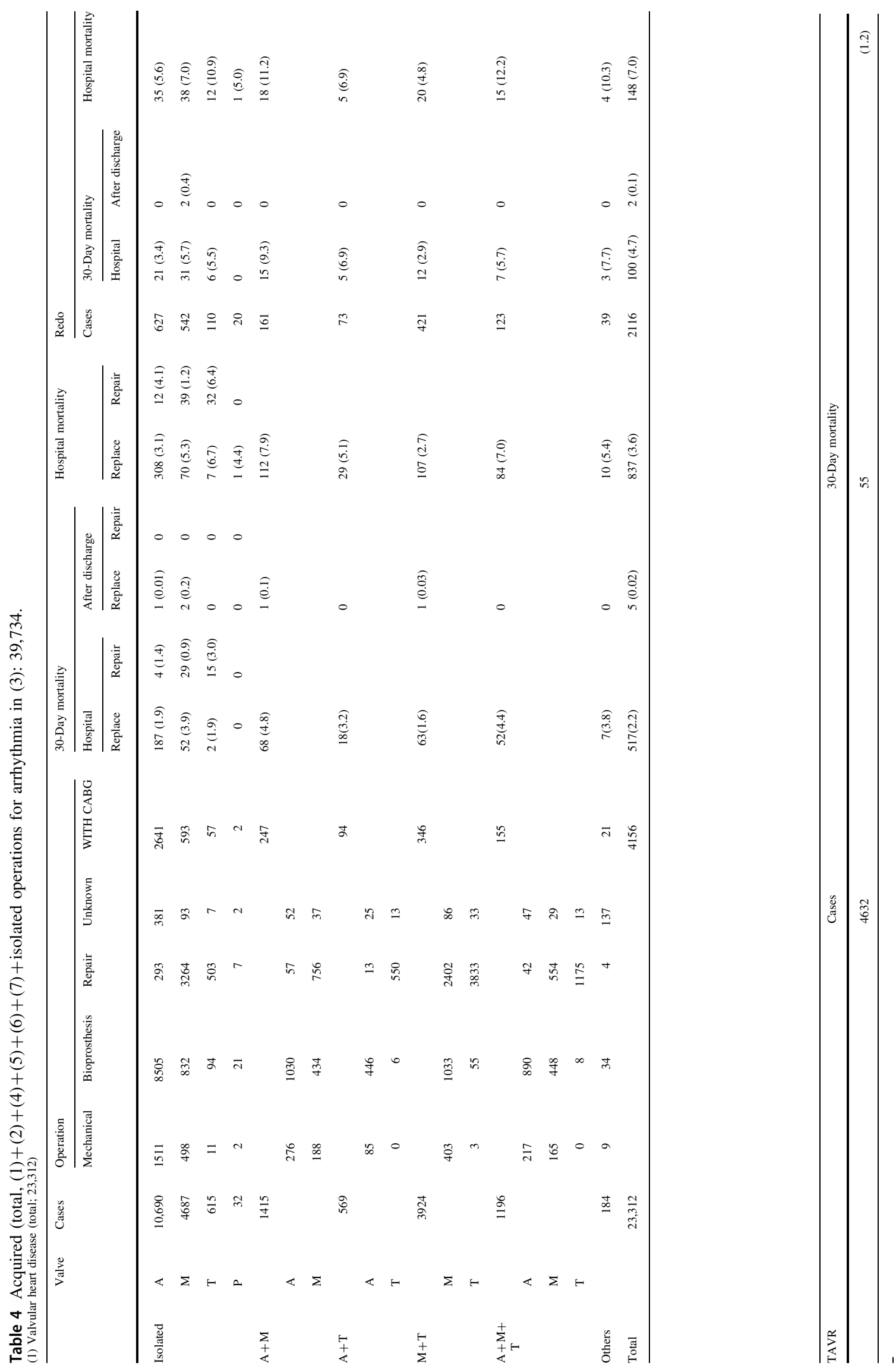




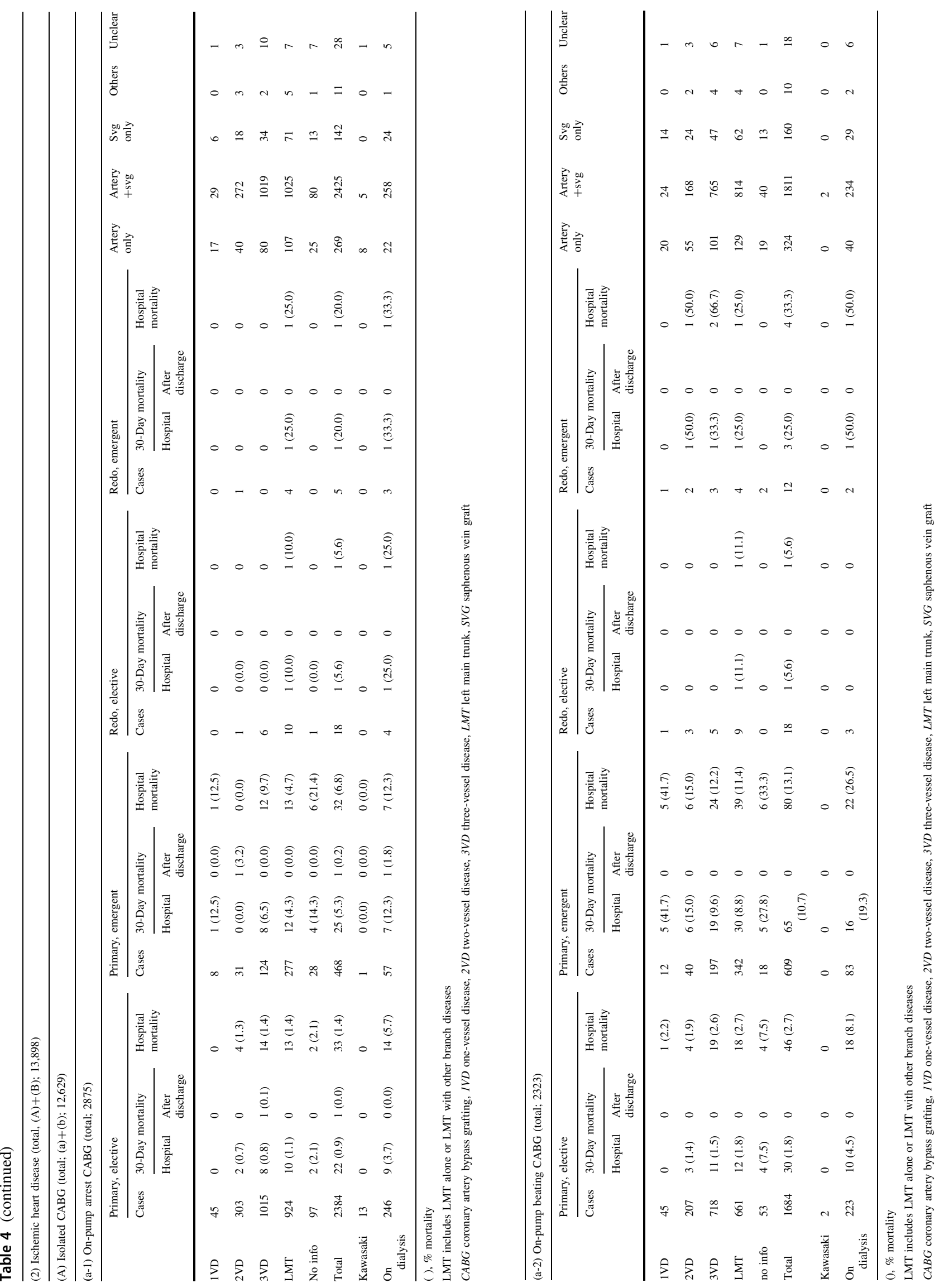




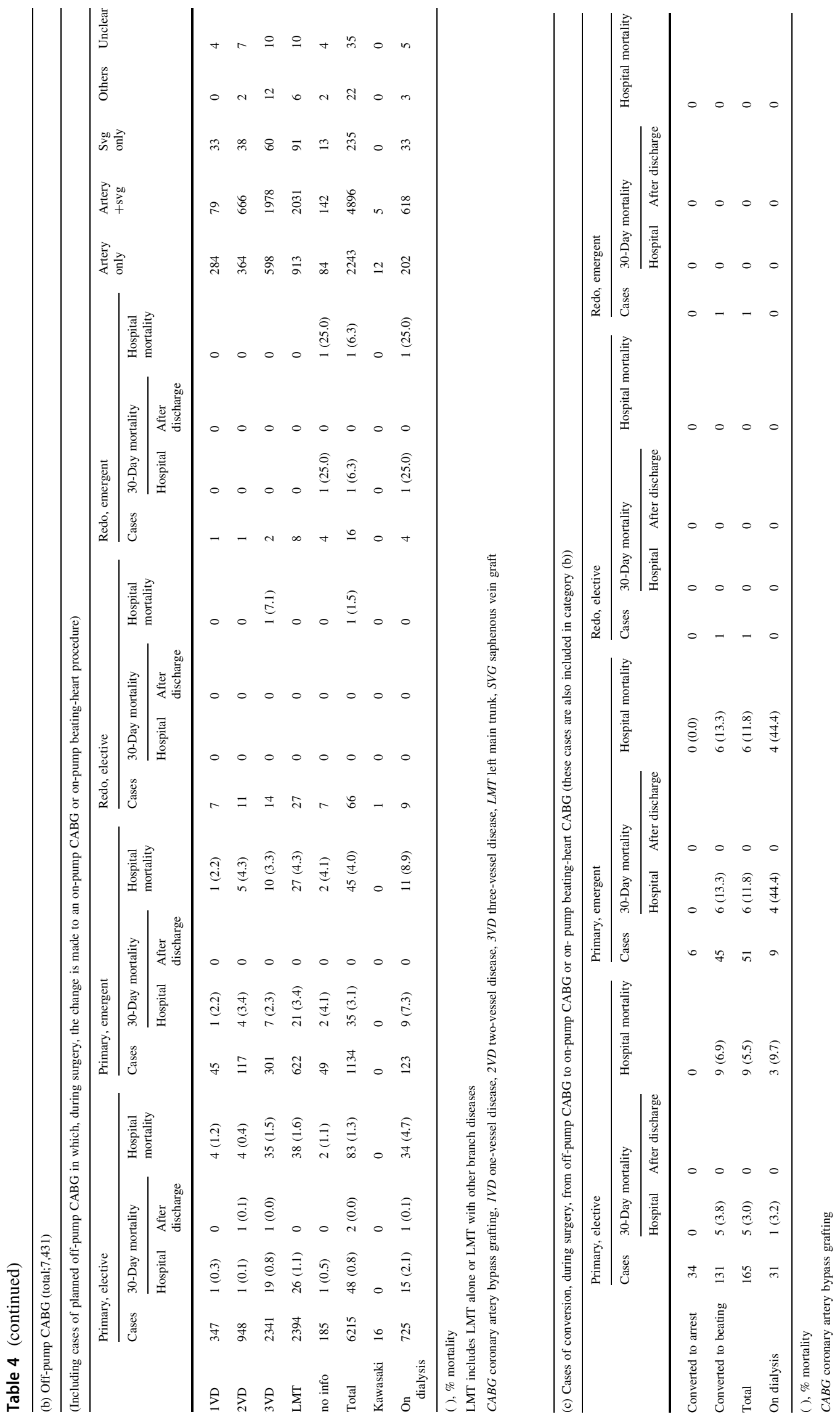




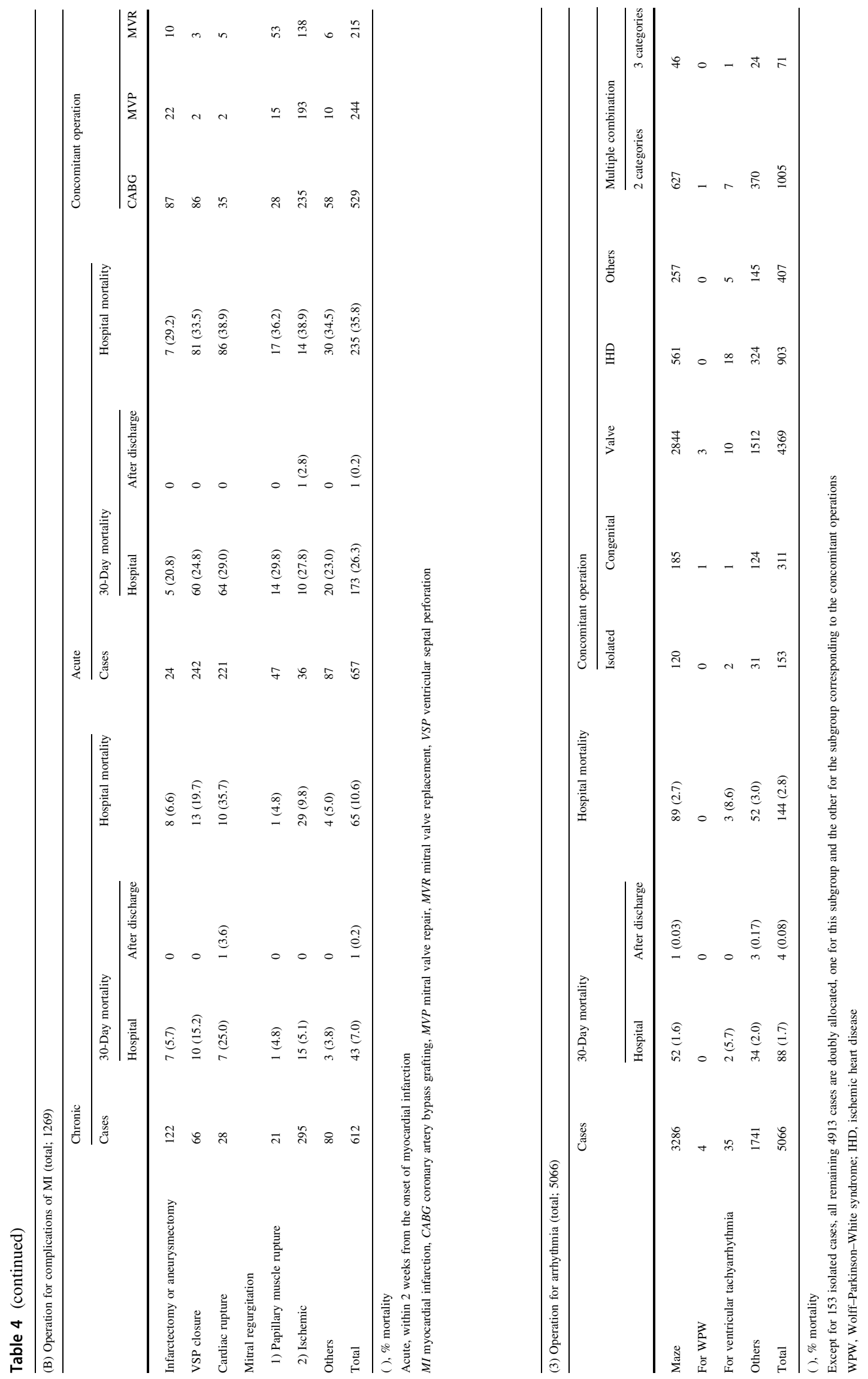




$$
\text { WF }
$$




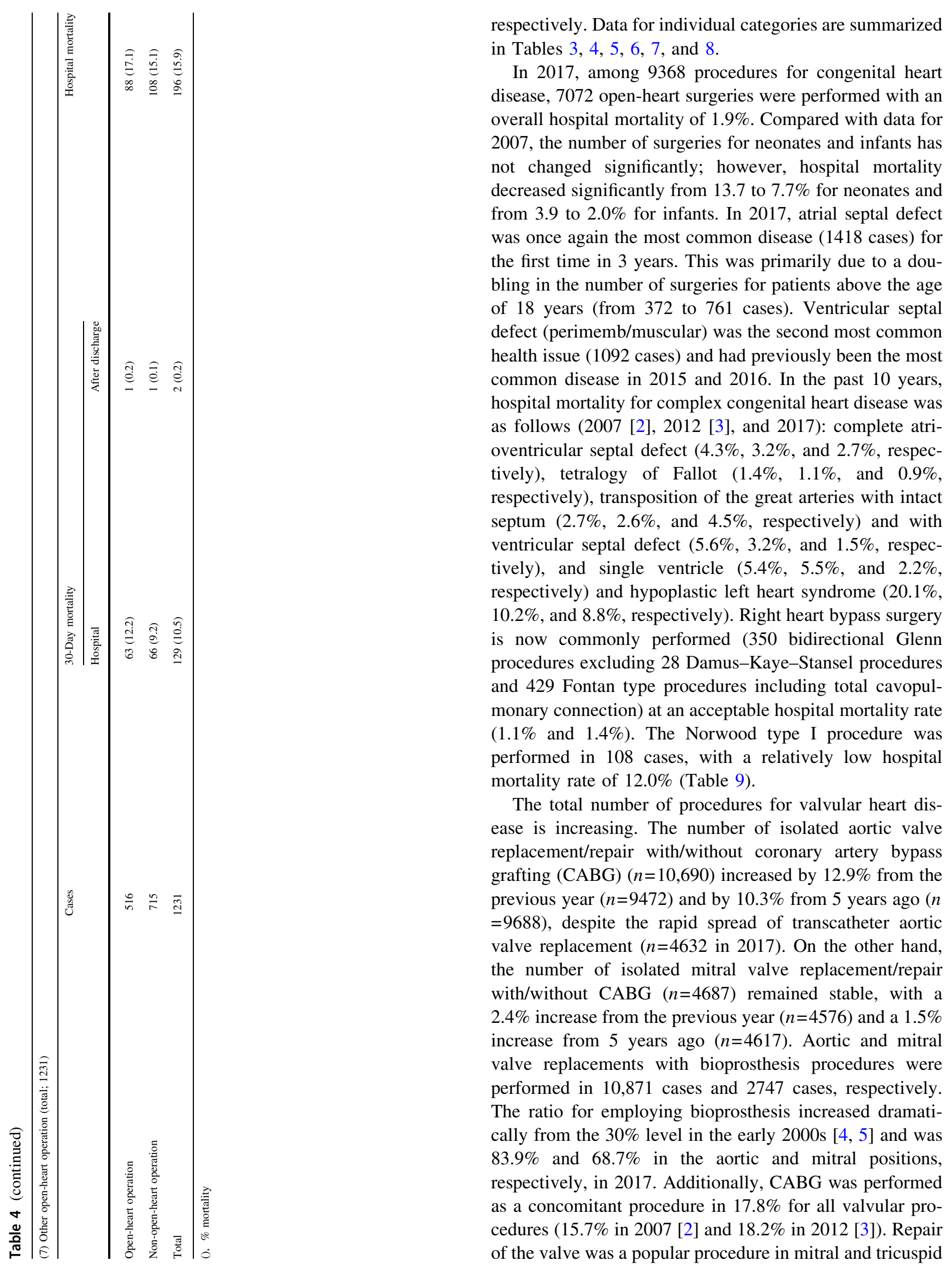


428

General Thoracic and Cardiovascular Surgery (2020) 68:414-449

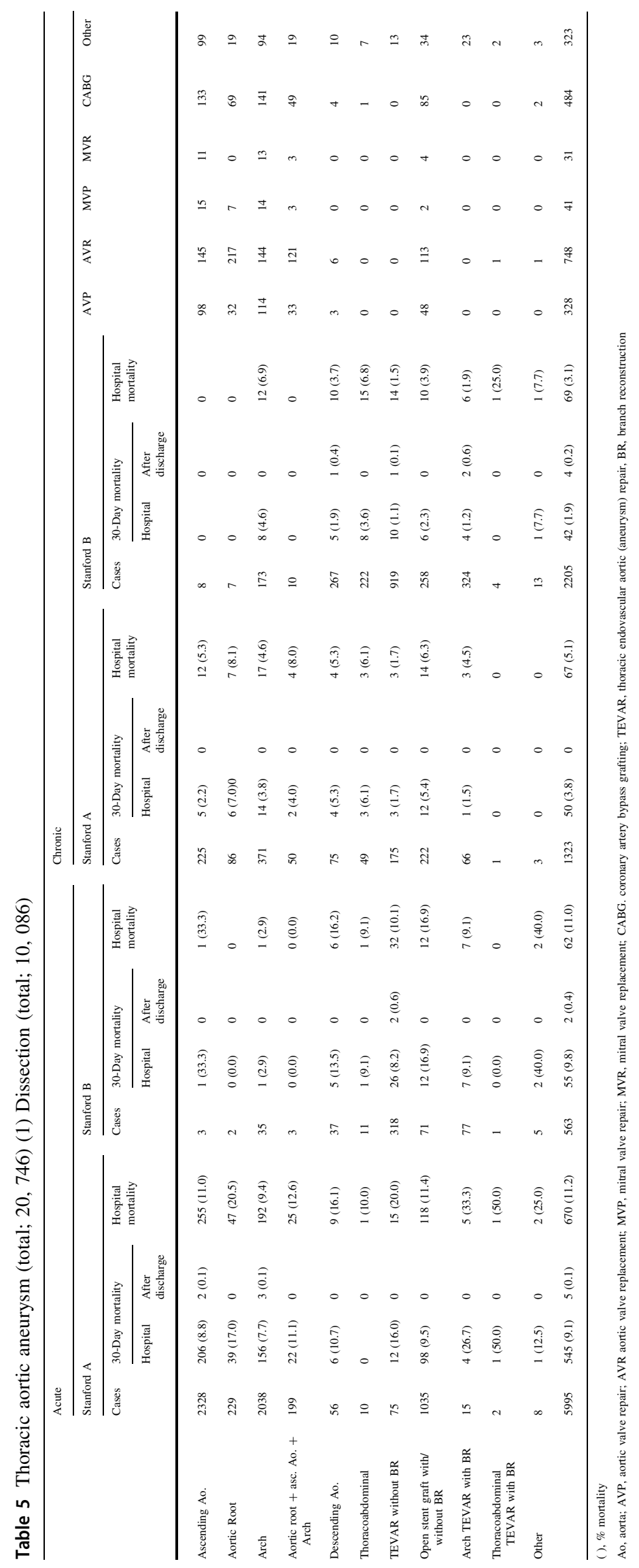

黑 Springer 


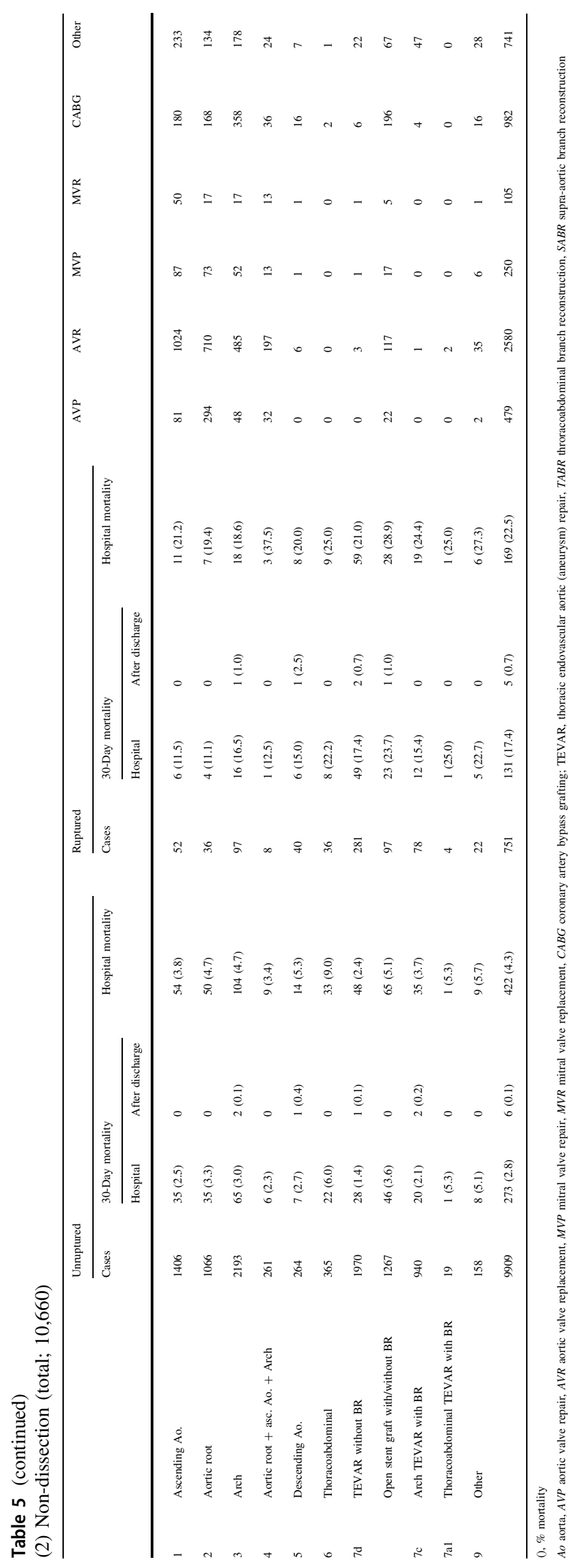


Table 6 Pulmonary thromboembolism (total; 174)

\begin{tabular}{lrlll}
\hline & Cases & \multicolumn{2}{l}{30 -Day mortality } & Hospital mortality \\
\cline { 3 - 4 } & & Hospital & After discharge \\
\hline Acute & 101 & $11(10.9)$ & 0 & $12(11.9)$ \\
Chronic & 73 & $3(4.1)$ & 0 & $7(9.6)$ \\
Total & 174 & $14(8.0)$ & 0 & $19(10.9)$ \\
\hline
\end{tabular}

(), \% mortality

Table 7 Implantation of VAD (total; 172)

\begin{tabular}{llll}
\hline & Cases & 30 -Day mortality & $\begin{array}{l}\text { Hospital } \\
\text { mortality }\end{array}$ \\
\cline { 3 - 4 } & & Hospital & $\begin{array}{l}\text { After } \\
\text { discharge }\end{array}$ \\
\hline $\begin{array}{l}\text { Implantation of } \\
\text { VAD }\end{array}$ & 172 & $5(2.9)$ & $7(4.1)$ \\
\hline
\end{tabular}

(), mortality \%

$V A D$ ventricular assist device

Table 8 Heart transplantation (total; 56)

\begin{tabular}{lllll}
\hline & Cases & \multicolumn{2}{l}{ 30-Day mortality } & $\begin{array}{l}\text { Hospital } \\
\text { mortality }\end{array}$ \\
\cline { 3 - 4 } & & Hospital & $\begin{array}{l}\text { After } \\
\text { discharge }\end{array}$ \\
\hline $\begin{array}{l}\text { Heart transplantation } \\
\begin{array}{l}\text { Heart and lung } \\
\text { transplantation }\end{array}\end{array}$ & 56 & $1(1.8)$ & 0 & $1(1.8)$ \\
Total & 56 & $1(1.8)$ & 0 & 0 \\
\hline
\end{tabular}

(), mortality \%

valve positions (6976 cases in the mitral and 6061 cases in the tricuspid) but is less frequently observed in aortic valve positions (405 patients, only $2.7 \%$ of all aortic valve procedures). Mitral valve repair constituted $29.9 \%$ of all valvular operations and $62.2 \%$ of all mitral valve procedures. Hospital mortality for single valve replacement was
$3.1 \%$ and $5.3 \%$ for the aortic and mitral positions, respectively, whereas for mitral valve repair, this was only $1.2 \%$. The hospital mortality for redo valve surgery was $3.4 \%$ and $5.7 \%$ in the aortic and mitral positions, respectively. Finally, overall hospital mortality did not show dramatic improvement during the past 10 years $3.8 \%$ in 2007 [2], 3.2\% in 2012 [3], and 3.6\% in 2017).

Isolated CABG was performed in 12,629 cases, representing only $73.0 \%$ of the number performed 10 years ago $(n=17,295)$ [2]. Among these, off-pump CABG was intended in 7431 cases $(58.8 \%)$ at a success rate of $97.1 \%$. The percentage of intended off-pump CABG in 2017 was less than $60 \%$ for the first time in 13 years, since 2004 [4]. Hospital mortality associated with primary elective CABG procedures in 10,283 cases was $1.6 \%$, unchanged from 2003 (1.5\%) [5]. Hospital mortality for primary emergency CABG in 2211 cases was still as high as $7.1 \%$. The result of conversion from off-pump CABG was $2.9 \%$, and hospital mortality in this context was $6.9 \%$. Hospital mortality was higher in patients with end-stage renal failure on dialysis, regardless of surgical procedures (on-pump arrest, on-pump beating, and off-pump). In this report, the number of concomitant CABGs alongside other major procedures was not included in the category of ischemic heart disease but in other categories such as valvular heart disease and thoracic aortic aneurysm. Accordingly, the overall number of CABGs, including concomitant CABG with other major procedures, remained more than 18,000 cases per year (18,327 cases) in 2017.

Measures for arrhythmia were performed primarily as concomitant procedures in 5066 cases, with hospital mortality of $2.8 \%$. Implantation of pacemaker and implantable cardioverter-defibrillator was not included in this category.

In $2017,20,746$ procedures were performed for thoracic and thoracoabdominal aortic diseases; 10,086 and 10,660 were for aortic dissection and non-dissection, respectively. The number of surgeries for aortic dissection increased by $6.9 \%$ this year, compared with that in the preceding year $(n$ =9441). The hospital mortality of procedures for 5995 Stanford type A acute aortic dissections remained as high as $11.2 \%$. The number of procedures for non-dissected aneurysm increased by $10.6 \%$, with overall hospital mortality of $5.5 \%$, and $4.3 \%$ and $22.5 \%$ for unruptured and ruptured aneurysms, respectively. The rate of thoracic endovascular aortic repair (TEVAR) among all operative 
procedures for aortic diseases is increasing. A total of 3563 patients with aortic dissection underwent stent graft placement: 1969 TEVARs and 1594 open stent graftings, respectively. The number of TEVARs for type B chronic aortic dissections was 1505 cases and accounted for $68.3 \%$ of total cases. The hospital mortality rates associated with TEVAR for type B aortic dissection were $11.0 \%$ and $3.1 \%$ in acute and chronic cases, respectively. A total of 4656 patients with non-dissected aortic aneurysm underwent stent graft placement, comprising 3269 TEVARs (a 6.7\% increase compared with that in 2016, $n=3063$ ) and 1387 open stent graftings (a 16.2\% increase compared with that in 2016, $n=1194)$. The hospital mortality rates for TEVARs and open stenting were as follows: TEVAR, $2.8 \%$ and $21.7 \%$ for unruptured and ruptured aneurysms, respectively, and open stent grafting, $5.1 \%$ and $28.7 \%$ for unruptured and ruptured aneurysms, respectively.

\section{(B) General thoracic surgery}

The 2017 survey of general thoracic surgery comprised 678 surgical units, with the bulk of data submitted via a webbased collection system of the NCD [1]. In total, 85,307 procedures were reported by general thoracic surgery departments in 2017, twice the number of operations in 2000 and 12,560 more than in 2012 (Fig. 2).

In $2017,44,140$ procedures for primary lung cancer were performed, a number that has increased annually. The 2017 value was 2.4 times that of 2000. Procedures for lung cancer represented $52 \%$ of all general thoracic surgery instances.

The number of video-assisted thoracoscopic surgery (VATS) instances, defined by a surgical procedure utilizing a skin incision longer than $8 \mathrm{~cm}$ and/or a minithoracotomy (hybrid) approach, has been noted since the 2015 annual report. The number of VATS procedures for benign pulmonary tumors and primary lung cancer, and the total number of VATS procedures in 2016 are shown in
Table 9 Total cases of general thoracic surgery during 2017

\begin{tabular}{lrr}
\hline & Cases & \multicolumn{1}{c}{$\%$} \\
\hline Benign pulmonary tumor & 2197 & 2.6 \\
Primary lung cancer & 44,140 & 51.7 \\
Other primary malignant pulmonary tumor & 423 & 0.5 \\
Metastatic pulmonary tumor & 8950 & 10.5 \\
Tracheal tumor & 120 & 0.1 \\
Mesothelioma & 698 & 0.8 \\
Chest wall tumor & 691 & 0.8 \\
Mediastinal tumor & 5197 & 6.1 \\
Thymectomy for MG without thymoma & 189 & 0.2 \\
Inflammatory pulmonary disease & 2423 & 2.8 \\
Empyema & 2962 & 3.5 \\
Bullous disease excluding pneumothorax & 400 & 0.5 \\
Pneumothorax & 14,499 & 17.0 \\
Chest wall deformity & 193 & 0.2 \\
Diaphragmatic hernia including traumatic & 28 & 0.0 \\
Chest trauma excluding diaphragmatic hernia & 443 & 0.5 \\
Lung transplantation & 68 & 0.1 \\
Others & 1686 & 2.0 \\
Total & 85,307 & 100.0 \\
\hline & &
\end{tabular}

Tables 10, 11, 13, 16, 17, 18, 19, 20, 21, 22, 23, 25, 26, and 27 , respectively.

In 2017, 2197 procedures were conducted for benign pulmonary tumors (Table 10), a similar number to that recorded in 2016. Hamartoma was the most frequent diagnosis in procedures for benign pulmonary tumors. VATS was performed for 2071 patients (94\%).

Additional information on primary malignant pulmonary tumors is shown in Tables 11 and 12 . With regard to lung cancer sub-type, adenocarcinoma was by far the most frequent diagnosis ( $71 \%$ of all lung cancer procedures), followed by squamous cell carcinoma (18\%). Sublobar resection was performed in 11,784 lung cancer cases (27\% of all cases) and lobectomy in 31,584 cases ( $72 \%$ of

General Thoracic Surgery

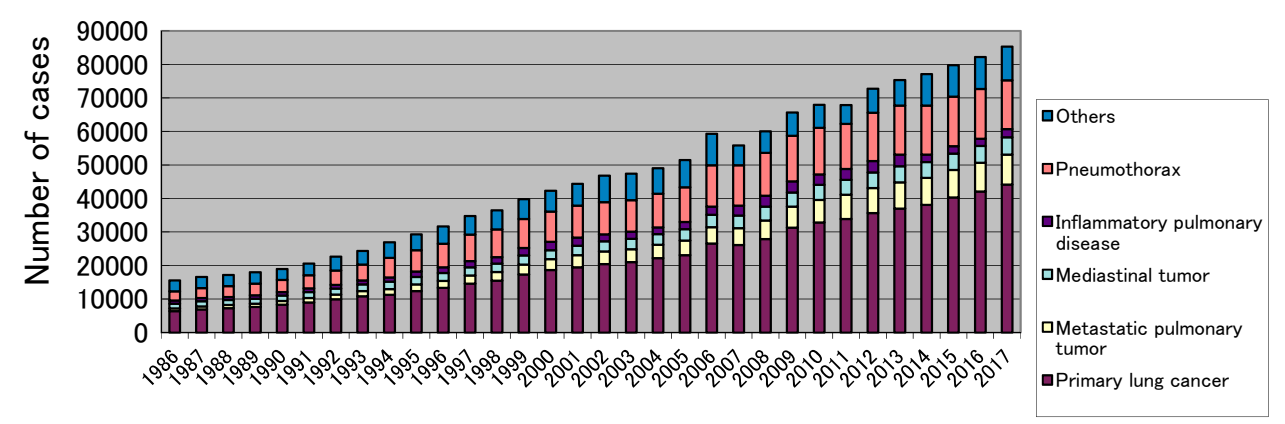

Calendar Year

Fig. 2 General thoracic surgery 
Table 10 Benign pulmonary tumor

\begin{tabular}{|c|c|c|c|c|c|}
\hline & \multirow[t]{2}{*}{ Cases } & \multicolumn{2}{|c|}{ 30-Day mortality } & \multirow{2}{*}{$\begin{array}{l}\text { Hospital } \\
\text { mortality }\end{array}$} & \multirow{2}{*}{$\begin{array}{l}\text { By } \\
\text { VAT }\end{array}$} \\
\hline & & Hospital & $\begin{array}{l}\text { After } \\
\text { discharge }\end{array}$ & & \\
\hline \multicolumn{6}{|c|}{ Benign pulmonary tumor } \\
\hline Hamartoma & 518 & 0 & 0 & 0 & 498 \\
\hline $\begin{array}{l}\text { Sclerosing } \\
\text { hemangioma }\end{array}$ & 76 & 0 & 0 & 0 & 73 \\
\hline Papilloma & 23 & 0 & 0 & 0 & 21 \\
\hline $\begin{array}{l}\text { Mucous gland } \\
\text { adenoma } \\
\text { bronchial }\end{array}$ & 2 & 0 & 0 & 0 & 2 \\
\hline Fibroma & 132 & 0 & 0 & 0 & 129 \\
\hline Lipoma & 4 & 0 & 0 & 0 & 4 \\
\hline Neurogenic tumor & 21 & 0 & 0 & 0 & 17 \\
\hline Clear cell tumor & 3 & 0 & 0 & 0 & 3 \\
\hline Leiomyoma & 18 & 0 & 0 & 0 & 17 \\
\hline Chondroma & 7 & 0 & 0 & 0 & 7 \\
\hline $\begin{array}{l}\text { Inflammatory } \\
\text { myofibroblastic } \\
\text { tumor }\end{array}$ & 5 & 0 & 0 & 0 & 5 \\
\hline Pseudolymphoma & 19 & 0 & 0 & 0 & 17 \\
\hline Histiocytosis & 13 & 0 & 0 & 0 & 12 \\
\hline Teratoma & 6 & 0 & 0 & 0 & 2 \\
\hline Others & 1350 & $2(0.1)$ & $2(0.1)$ & $5(0.4)$ & 1264 \\
\hline Total & 2197 & $2(0.1)$ & $2(0.09)$ & $5(0.2)$ & 2071 \\
\hline
\end{tabular}

(), mortality \%

all cases). Sleeve lobectomy was performed in 496 cases, and pneumonectomy was required in 403 cases $(0.9 \%$ of all cases). VATS lobectomy for lung cancer was performed in 21,992 cases ( $70 \%$ of all lobectomy cases). The number of VATS procedures for primary lung cancer was slightly higher in 2017 than that in 2016. The number of the patients aged 80 years or older who underwent lung cancer surgery was $5779(13 \%)$. In total, 121 patients died prior to hospital discharge within 30 days following surgery, and 32 patients died following discharge within 30 days after surgery. Therefore, 153 patients died within 30 days after surgery (30-day mortality rate $0.3 \%$ ). In total, 247 patients died prior to discharge (hospital mortality rate $0.6 \%$ ), and the 30-day mortality rate, according to procedure, was $0.2 \%$ for segmentectomy, $0.3 \%$ for lobectomy, and $2 \%$ for pneumonectomy. Interstitial pneumonia was the leading cause of death following lung cancer surgery, followed by pneumonia, respiratory failure, and cardiovascular events.

Procedures for metastatic pulmonary tumors, 8950 of which were performed in 2017, are shown in Table 13. In this instance, colorectal cancer was the most frequent diagnosis ( $47 \%$ of all cases).
Table 11 Primary malignant pulmonary tumor

\begin{tabular}{|c|c|c|c|c|c|}
\hline & \multirow[t]{2}{*}{ Cases } & \multicolumn{2}{|c|}{ 30-Day mortality } & \multirow{2}{*}{$\begin{array}{l}\text { Hospital } \\
\text { mortality }\end{array}$} & \multirow[t]{2}{*}{ VATS } \\
\hline & & Hospital & $\begin{array}{l}\text { After } \\
\text { discharge }\end{array}$ & & \\
\hline Primary malignant pulmonary tumor & 44,563 & $\begin{array}{l}122 \\
(0.3)\end{array}$ & $32(0.1)$ & $247(0.6)$ & 32,206 \\
\hline Lung cancer & 44,140 & $\begin{array}{l}121 \\
(0.3)\end{array}$ & $31(0.1)$ & $244(0.6)$ & 32,206 \\
\hline Adenocarcinoma & 31,119 & $52(0.2)$ & $13(0.0)$ & $91(0.3)$ & \\
\hline Squamous cell carcinoma & 8132 & $56(0.7)$ & $14(0.2)$ & $121(1.5)$ & \\
\hline Large cell carcinoma & 301 & $1(0.3)$ & $1(0.3)$ & $4(1.3)$ & \\
\hline LCNEC & 601 & $3(0.5)$ & 0 & $4(0.7)$ & \\
\hline Small cell carcinoma & 823 & $2(0.2)$ & 0 & $4(0.5)$ & \\
\hline Adenosquamous carcinoma & 571 & $1(0.2)$ & $2(0.4)$ & $2(0.4)$ & \\
\hline $\begin{array}{l}\text { Carcinoma with pleomorphic, } \\
\text { sarcomatoid or sarcomatous } \\
\text { elements }\end{array}$ & 493 & $2(0.4)$ & 0 & $9(1.8)$ & \\
\hline Carcinoid & 233 & 0 & 0 & 0 & \\
\hline Carcinomas of salivary-gland type & 38 & 0 & 0 & $1(2.6)$ & \\
\hline Unclassified & 43 & 0 & 0 & 0 & \\
\hline Multiple lung cancer & 1464 & $3(0.2)$ & $1(0.1)$ & $6(0.4)$ & \\
\hline Others & 322 & $1(0.3)$ & 0 & $2(0.6)$ & \\
\hline Wedge resection & 7099 & $14(0.2)$ & $8(0.1)$ & $28(0.4)$ & 6299 \\
\hline Segmental excision & 4685 & $9(0.19)$ & $2(0.0)$ & $16(0.3)$ & 3698 \\
\hline (Sleeve segmental excision) & 14 & 0 & 0 & 0 & 3 \\
\hline Lobectomy & 31,584 & $85(0.3)$ & $18(0.1)$ & $179(0.6)$ & 21,992 \\
\hline (Sleeve lobectomy) & 496 & $1(0.2)$ & $2(0.4)$ & $2(0.4)$ & 71 \\
\hline Pneumonectomy & 403 & $8(2.0)$ & $1(0.2)$ & $15(3.7)$ & 33 \\
\hline (Sleeve pneumonectomy) & 10 & $2(20.0)$ & 0 & $2(20.0)$ & 2 \\
\hline Other bronchoplasty & 33 & 0 & 0 & 0 & 4 \\
\hline Pleuropneumonectomy & 1 & 0 & 0 & 0 & 0 \\
\hline Others & 335 & $5(1.5)$ & $2(0.6)$ & $6(1.8)$ & 180 \\
\hline Unknown & 0 & 0 & 0 & 0 & \\
\hline Sarcoma & 56 & $1(1.8)$ & $1(1.8)$ & $1(1.8)$ & \\
\hline AAH & 117 & 0 & 0 & 0 & \\
\hline Others & 250 & 0 & 0 & $2(0.8)$ & \\
\hline
\end{tabular}

(), mortality \%

There were 46 procedures for malignant tracheal tumor in 2017; however, 21 patients were treated with sleeve resection and reconstruction (Table 14).

Pleural tumors numbered 698 in 2017 (Table 15). Diffuse malignant pleural mesothelioma was the most frequent histologic diagnosis. Total pleurectomy was performed in 104 cases and extrapleural pneumonectomy in 65 cases. The 30-day mortality rate was $0 \%$ following total pleurectomy and $2 \%$ after extrapleural pneumonectomy, both representing better outcomes than before.

In total, 691 chest wall tumors were resected in 2017 (Table 16), $362(52 \%)$ of which were benign. Among the 329 malignant chest wall tumors, 189 (57\%) were metastatic tumors. 
Table 12 Details of lung cancer operations

\begin{tabular}{lr}
\hline TNM & \\
\hline c-Stage & Cases \\
\hline IA1 & 7464 \\
IA2 & 12,312 \\
IA3 & 7771 \\
IB & 5228 \\
IIA & 1661 \\
IIB & 3846 \\
IIIA & 2802 \\
IIIB & 524 \\
IIIC & 25 \\
IVA & 449 \\
IVB & 70 \\
NA & 1988 \\
Total & 44,140 \\
\hline Sex & Cases \\
\hline Male & 27,089 \\
Female & 14,051 \\
NA & 0 \\
Total & 41,140 \\
\hline Case & \\
\hline
\end{tabular}

\begin{tabular}{lr}
\hline Cause of death & Case \\
\hline Cardiovascular & 23 \\
Pneumonia & 43 \\
Pyothorax & 2 \\
Bronchopleural fistula & 11 \\
Respiratory failure & 25 \\
Pulmonary embolism & 5 \\
Interstitial pneumonia & 78 \\
Brain infarction or bleeding & 11 \\
Others & 62 \\
Unknown & 15 \\
Total & 275 \\
\hline
\end{tabular}

\begin{tabular}{lr}
\hline p-Stage & Cases \\
\hline O(pCR) & 2940 \\
IA1 & 8908 \\
IA2 & 9422 \\
IA3 & 4873 \\
IB & 5999 \\
IIA & 1242 \\
IIB & 4579 \\
IIIA & 4026 \\
IIIB & 818 \\
IIIC & 17 \\
IVA & 982 \\
IVB & 69 \\
NA & 265 \\
\hline
\end{tabular}

Table 12 continued

\begin{tabular}{lr}
\hline p-Stage & Cases \\
\hline Total & 44,140 \\
\hline Age (years) & Cases \\
\hline$<20$ & 20 \\
$20-29$ & 38 \\
$30-39$ & 209 \\
$40-49$ & 1226 \\
$50-59$ & 3768 \\
$60-69$ & 14,080 \\
$70-79$ & 19,020 \\
$80-89$ & 5681 \\
$\geq 90$ & 98 \\
NA & 0 \\
Total & 44,140 \\
\hline
\end{tabular}

Mediastinal tumors were resected in 5197 patients in 2017, a slight increase from the previous year (Table 17). Thymic epithelial tumor-including 1939 thymomas, 368 thymic carcinomas, and 39 thymic carcinoids-was the most frequent mediastinal tumor type in 2017.

Thymectomy for myasthenia gravis was performed in 508 patients (Table 18); 319 procedures were associated with thymoma, and the remaining was not associated with thymoma.

Procedures for non-neoplastic disease were performed for 22,634 patients. There were 2423 cases of lung resection for inflammatory lung diseases (Table 19), $20 \%$ of which were associated with atypical mycobacterium infections and $15 \%$ with fungal infections. Procedures for inflammatory nodules were performed in cases where lung cancer was suspected prior to surgery (in 918 cases, 38\%).

The 2962 procedures for empyema (Table 20) comprised 2226 cases $(75 \%)$ of acute empyema and 736 cases of chronic empyema. Bronchopleural fistula was reported in 453 patients with acute empyema and in 355 patients with chronic empyema. The hospital mortality rate was $15 \%$ among patients with acute empyema with fistula.

In 2017, 101 operations were performed for descending necrotizing mediastinitis (Table 21). The hospital mortality rate was $7 \%$. Furthermore, 400 procedures were conducted for bullous diseases (Table 22); lung volume reduction surgery was performed in only 28 patients.

A total of 14,499 procedures were performed for spontaneous pneumothorax (Table 23). The 11,113 procedures for primary pneumothorax comprised 2838 patients (26\%) who underwent bullectomy only and 7488 patients (67\%) who underwent an additional procedure. There were 3386 
Table 13 Metastatic pulmonary tumor

Table 14 Tracheal tumor

\begin{tabular}{llllll}
\hline & Cases & \multicolumn{2}{l}{30 -Day mortality } & Hospital mortality & \multirow{2}{*}{ VATS } \\
\cline { 3 - 4 } & & Hospital & After discharge & \\
\hline Metastatic pulmonary tumor & 8950 & $12(0.1)$ & $4(0.0)$ & $18(0.2)$ & 8298 \\
Colorectal & 4240 & $5(0.1)$ & 0 & $7(0.2)$ & 3965 \\
Hepatobiliary/pancreatic & 422 & 0 & 0 & $1(0.2)$ & 400 \\
Uterine & 448 & 0 & 0 & 0 & 425 \\
Mammary & 545 & 0 & $1(0.2)$ & 0 & 517 \\
Ovarian & 71 & 0 & 0 & 0 & 65 \\
Testicular & 72 & $1(1.4)$ & $1(1.4)$ & $1(1.4)$ & 66 \\
Renal & 746 & 0 & 0 & 0 & 706 \\
Skeletal & 144 & 0 & 0 & $1(0.7)$ & 124 \\
Soft tissue & 274 & 0 & $1(0.4)$ & 0 & 248 \\
Otorhinolaryngological & 435 & 0 & 0 & 0 & 397 \\
Pulmonary & 573 & $3(0.5)$ & 0 & $4(0.7)$ & 483 \\
Others & 980 & $3(0.3)$ & $1(0.1)$ & $4(0.4)$ & 902 \\
\hline
\end{tabular}

(), mortality \%

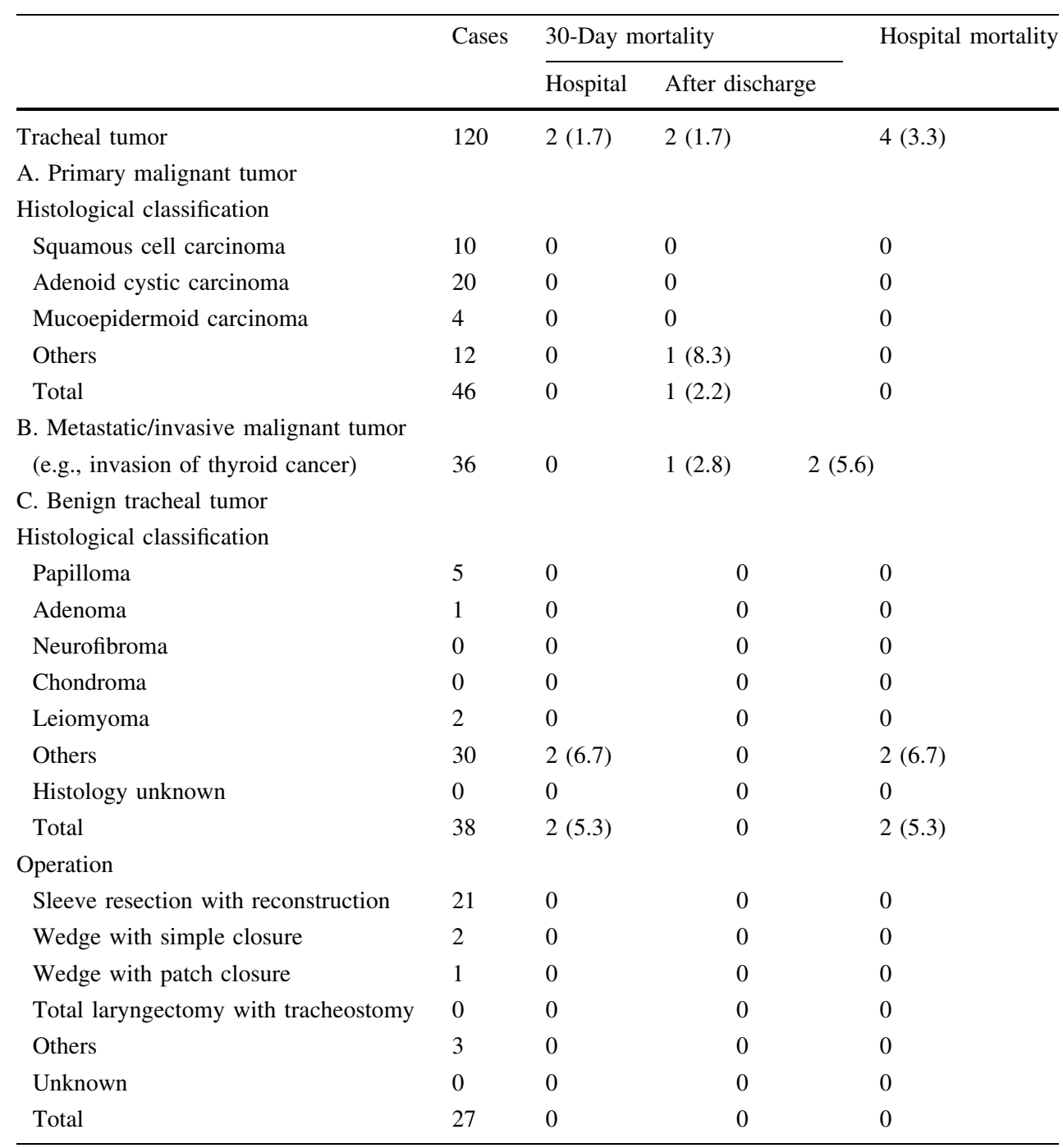

(), mortality \% 
Table 15 Tumor of pleural origin

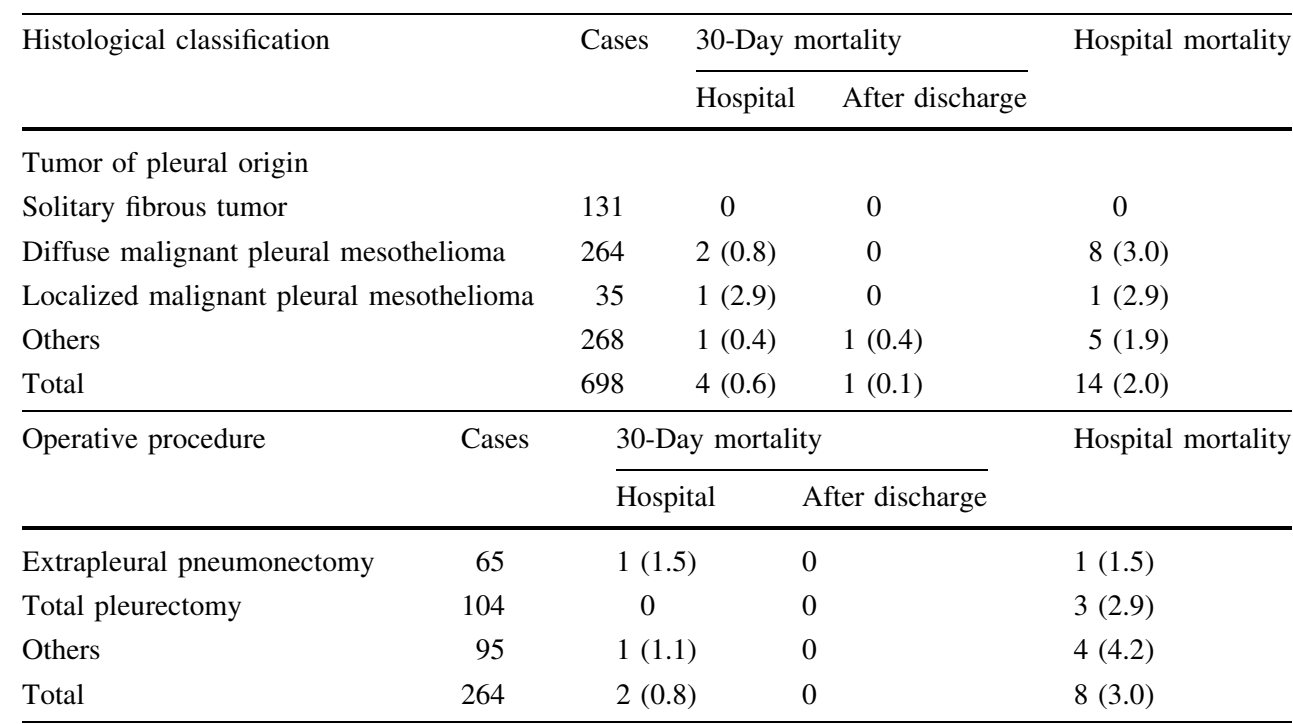

(), mortality \%

procedures for secondary pneumothorax, where COPD was by far the most prevalent associated disease (69\%). The hospital mortality rate for secondary pneumothorax associated with COPD was $2.6 \%$.

The 2017 survey reported 193 procedures for chest wall deformity (Table 24). However, this may have been underestimated, because the Nuss procedure for pectus excavatum was more likely to have been performed in pediatric surgery centers not associated with the Japanese Association for Thoracic Surgery.

Diaphragmatic hernia was treated surgically in 28 patients (Table 25). This figure may also have been underestimated, as procedures may have been classified as gastrointestinal surgery.
The survey reported 443 procedures for chest trauma excluding iatrogenic injuries (Table 26). In this context, hospital mortality rate was $5 \%$.

Table 27 shows procedures for other diseases, including 89 cases of arteriovenous malformation and 92 cases of pulmonary sequestration.

A total of 68 lung transplantations were performed in 2017 (Table 28): 57 patients received lung transplants from brain-dead donors, and 11 patients received transplants from living, related donors.

The number of VATS procedures has increased annually to reach 68,458 ( $80 \%$ of all general thoracic surgeries) in 2017 (Table 29).

The details of tracheobronchoplasty, pediatric surgery, and combined resection of neighboring organs are shown in Tables 30, 31, 32, and 33.

Table 16 Chest wall tumor

\begin{tabular}{lccccc}
\hline & Cases & \multicolumn{2}{l}{30 -Day mortality } & Hospital mortality & VATS \\
\cline { 3 - 4 } & & Hospital & After discharge & \\
\hline Chest wall tumor & & & & \\
Primary malignant tumor & 140 & $1(0.7)$ & $1(0.7)$ & $6(4.3)$ & 65 \\
Metastatic malignant tumor & 189 & 0 & $1(0.5)$ & 0 & 77 \\
Benign tumor & 362 & $1(0.3)$ & 0 & $1(0.3)$ & 281 \\
Total & 691 & $2(0.3)$ & $2(0.3)$ & $7(1.0)$ & 423 \\
\hline
\end{tabular}

(), mortality \% 
Table 17 Mediastinal tumor

\begin{tabular}{|c|c|c|c|c|c|}
\hline & \multirow[t]{2}{*}{ Cases } & \multicolumn{2}{|c|}{ 30-Day mortality } & \multirow[t]{2}{*}{ Hospital mortality } & \multirow[t]{2}{*}{ By VATS } \\
\hline & & Hospital & After discharge & & \\
\hline Mediastinal tumor & 5197 & $3(0.1)$ & $3(0.06)$ & $9(0.2)$ & 3808 \\
\hline Thymoma* & 1939 & $1(0.1)$ & $2(0.1)$ & $3(0.2)$ & 1222 \\
\hline Thymic cancer & 368 & 0 & $1(0.3)$ & $1(0.3)$ & 189 \\
\hline Thymus carcinoid & 39 & 0 & 0 & 0 & 19 \\
\hline Germ cell tumor & 85 & 0 & 0 & 0 & 59 \\
\hline Benign & 66 & 0 & 0 & 0 & 51 \\
\hline Malignant & 19 & 0 & 0 & 0 & 8 \\
\hline Neurogenic tumor & 489 & 0 & 0 & 0 & 447 \\
\hline Congenital cyst & 1185 & $1(0.1)$ & 0 & $1(0.1)$ & 1071 \\
\hline Goiter & 68 & 0 & 0 & 0 & 29 \\
\hline Lymphatic tumor & 185 & 0 & 0 & $1(0.5)$ & 134 \\
\hline Excision of pleural recurrence of thymoma & 27 & 0 & 0 & 0 & 20 \\
\hline Thymolipoma & 19 & 0 & 0 & 0 & 14 \\
\hline Others & 793 & $1(0.1)$ & 0 & $3(0.4)$ & 604 \\
\hline
\end{tabular}

(), mortality \%

Table 18 Thymectomy for myasthenia gravis with thymoma

\begin{tabular}{llllll}
\hline & Cases & \multicolumn{2}{l}{30 -Day mortality } & Hospital mortality & By VATS \\
\cline { 3 - 4 } & & Hospital & After discharge & \\
\hline Thymectomy for myasthenia & 508 & 0 & 0 & $1(0.2)$ & 298 \\
gravis with thymoma & 319 & 0 & 0 & $1(0.3)$ & 175 \\
\hline
\end{tabular}

(), mortality \%
Table 19 Operations for nonneoplastic diseases

\begin{tabular}{|c|c|c|c|c|c|c|c|c|}
\hline & \multirow{2}{*}{\multicolumn{2}{|c|}{ Cases }} & \multicolumn{4}{|c|}{ 30-Day mortality } & \multicolumn{2}{|c|}{ Hospital mortality } \\
\hline & & & \multicolumn{2}{|c|}{ Hospital } & \multicolumn{2}{|c|}{ After discharge } & & \\
\hline \multirow[t]{3}{*}{ Operations for non-neoplastic diseases } & \multicolumn{2}{|c|}{22,634} & \multicolumn{2}{|c|}{$214(0.9)$} & $31(0.1)$ & \multicolumn{3}{|c|}{$467(2.1)$} \\
\hline & \multirow[t]{2}{*}{ Cases } & \multicolumn{4}{|c|}{ 30-Day mortality } & \multirow{2}{*}{\multicolumn{2}{|c|}{ Hospital mortality }} & \multirow[t]{2}{*}{ VATS } \\
\hline & & \multicolumn{2}{|c|}{ Hospital } & \multicolumn{2}{|c|}{ After discharge } & & & \\
\hline A. Inflammatory pulmonary disease & 2423 & \multicolumn{2}{|c|}{$3(0.1)$} & \multicolumn{2}{|l|}{$2(0.1)$} & \multicolumn{2}{|c|}{$9(0.4)$} & 2165 \\
\hline Tuberculous infection & 46 & \multicolumn{2}{|c|}{0} & \multicolumn{2}{|l|}{0} & \multicolumn{2}{|l|}{0} & 41 \\
\hline Mycobacterial infection & 496 & \multicolumn{2}{|c|}{0} & \multicolumn{2}{|c|}{$1(0.2)$} & \multicolumn{2}{|c|}{$1(0.2)$} & 447 \\
\hline Fungal infection & 361 & \multicolumn{2}{|c|}{$1(0.3)$} & \multicolumn{2}{|l|}{0} & \multicolumn{2}{|c|}{$3(0.8)$} & 284 \\
\hline Bronchiectasis & 58 & \multicolumn{2}{|c|}{0} & \multicolumn{2}{|l|}{0} & \multicolumn{2}{|l|}{0} & 43 \\
\hline Tuberculous nodule & 79 & \multicolumn{2}{|c|}{0} & \multicolumn{2}{|l|}{0} & \multicolumn{2}{|l|}{0} & 70 \\
\hline Inflammatory pseudotumor & 918 & \multicolumn{2}{|c|}{0} & \multicolumn{2}{|l|}{$1(0.1)$} & 0 & & 858 \\
\hline Interpulmonary lymph node & 76 & \multicolumn{2}{|c|}{0} & \multicolumn{2}{|l|}{0} & 0 & & 74 \\
\hline Others & 389 & \multicolumn{2}{|c|}{$2(0.5)$} & 0 & & $5(1.3)$ & & 348 \\
\hline
\end{tabular}

(), mortality \% 
Table 20 B. Empyema

\begin{tabular}{|c|c|c|c|c|c|}
\hline & \multirow[t]{2}{*}{ Cases } & \multicolumn{2}{|c|}{ 30-Day mortality } & \multirow[t]{2}{*}{ Hospital mortality } & \multirow[t]{2}{*}{ By VATS } \\
\hline & & Hospital & After discharge & & \\
\hline Acute empyema & 2226 & $54(2.4)$ & $5(0.2)$ & $128(5.8)$ & 1858 \\
\hline With fistula & 453 & $26(5.7)$ & $1(0.2)$ & $69(15.2)$ & 251 \\
\hline Without fistula & 1756 & $27(1.5)$ & $4(0.2)$ & $57(3.2)$ & 1592 \\
\hline Unknown & 17 & $1(5.9)$ & 0 & $2(11.8)$ & 15 \\
\hline Chronic empyema & 736 & $20(2.7)$ & $3(0.4)$ & $58(7.9)$ & 406 \\
\hline With fistula & 355 & $9(2.5)$ & $2(0.6)$ & $23(6.5)$ & 148 \\
\hline Without fistula & 357 & $11(3.1)$ & $1(0.3)$ & $33(9.2)$ & 236 \\
\hline Unknown & 24 & 0 & 0 & $2(8.3)$ & 22 \\
\hline Total & 2962 & $74(2.5)$ & $8(0.3)$ & $186(6.3)$ & 2264 \\
\hline
\end{tabular}

(), mortality \%

Table 21 C. Descending necrotizing mediastinitis

\begin{tabular}{llllll}
\hline & Cases & \multicolumn{3}{l}{30 -Day mortality } & Hospital mortality \\
\cline { 3 - 5 } & & Hospital & After discharge & VATS \\
\hline C. Descending necrotizing mediastinitis & 101 & $5(5.0)$ & 0 & $75.9)$ \\
\hline (), mortality \% & & & & 75 \\
\hline
\end{tabular}

(), mortality \%

Table 22 D. Bullous diseases

\begin{tabular}{lcclcr}
\hline & Cases & \multicolumn{2}{l}{30 -Day mortality } & Hospital mortality & \multirow{2}{*}{ VATS } \\
\cline { 2 - 5 } & & Hospital & After discharge & \\
\hline D. Bullous diseases & 400 & $2(0.5)$ & 0 & $5(1.3)$ & 371 \\
Emphysematous bulla & 308 & $2(0.6)$ & 0 & $5(1.6)$ & 289 \\
Bronchogenic cyst & 13 & 0 & 0 & 0 & 12 \\
Emphysema with LVRS & 28 & 0 & 0 & 0 & 28 \\
Others & 51 & 0 & 0 & & 42 \\
\hline
\end{tabular}

(), mortality \%

LVRS lung volume reduction surgery 
Table 23 E. Pneumothorax

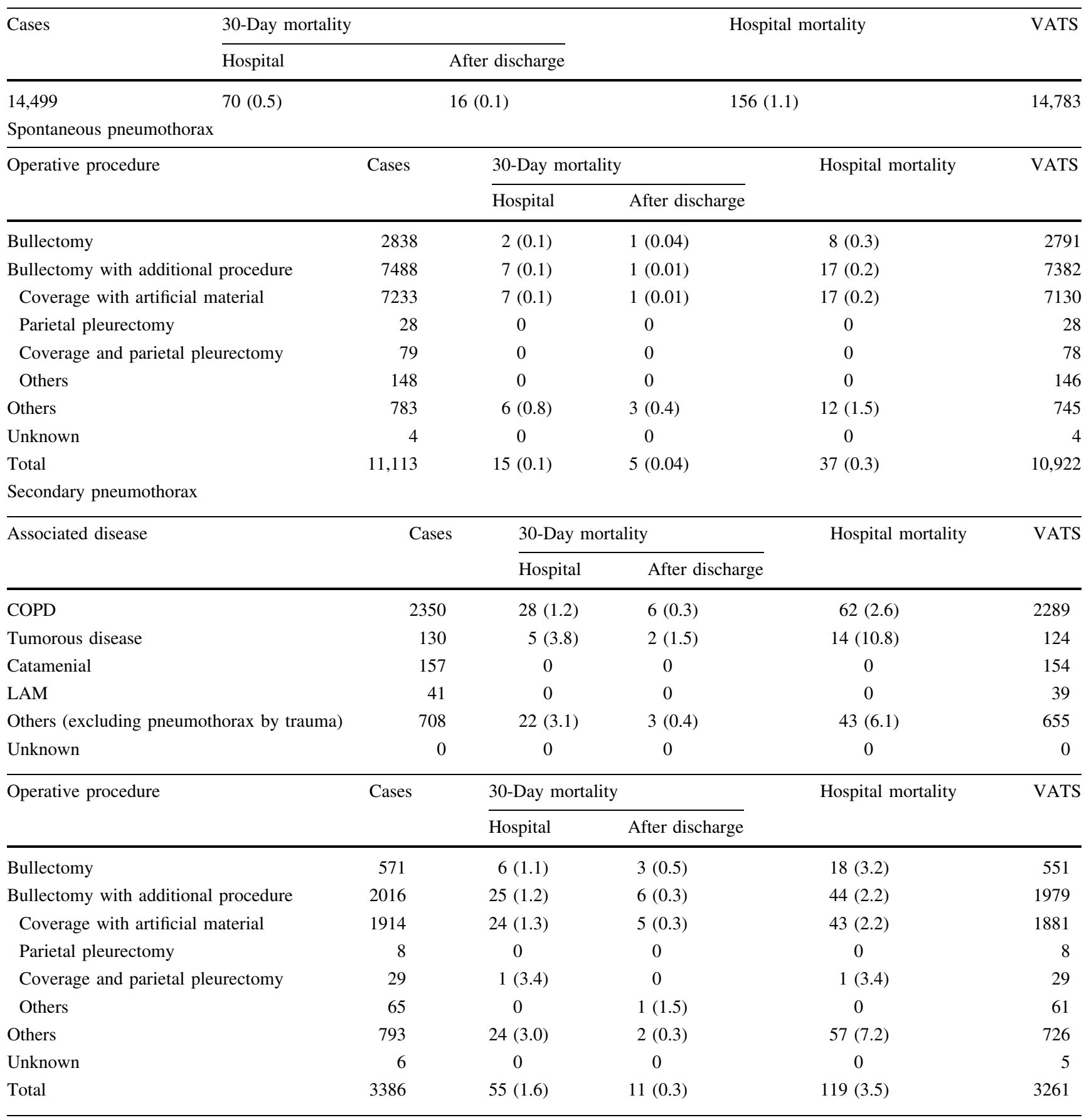

(), mortality \% 
Table 24 F. Chest wall deformity

\begin{tabular}{lrllc}
\hline & Cases & \multicolumn{2}{l}{ 30-Day mortality } & $\begin{array}{l}\text { Hospital } \\
\text { mortality }\end{array}$ \\
\cline { 3 - 4 } & & Hospital & $\begin{array}{l}\text { After } \\
\text { discharge }\end{array}$ & \\
\hline $\begin{array}{l}\text { F. Chest wall } \\
\text { deformity }\end{array}$ & 193 & $1(0.5)$ & 0 & $1(0.5)$ \\
$\begin{array}{l}\text { Funnel chest } \\
\text { Others }\end{array}$ & 182 & 0 & 0 & 0 \\
\hline
\end{tabular}

(), mortality \%

Table 25 G. Diaphragmatic hernia

\begin{tabular}{lccccc}
\hline & Cases & \multicolumn{2}{c}{ 30-Day mortality } & $\begin{array}{l}\text { Hospital } \\
\text { mortality }\end{array}$ & VATS \\
\cline { 3 - 5 } & \multicolumn{5}{c}{$\begin{array}{c}\text { Hospital } \\
\text { After } \\
\text { discharge }\end{array}$} \\
\hline $\begin{array}{l}\text { G. } \\
\text { Diaphragmatic } \\
\text { hernia }\end{array}$ & 28 & $1(3.6)$ & 0 & $1(3.6)$ & 19 \\
Congenital & 4 & 0 & 0 & 0 & 3 \\
Traumatic & 10 & 0 & 0 & 0 & 6 \\
Others & 14 & $1(7.1)$ & 0 & $1(7.1)$ & 10 \\
\hline
\end{tabular}

(), mortality \%

Table 26 H. Chest trauma

\begin{tabular}{|c|c|c|c|c|c|}
\hline & \multirow[t]{2}{*}{ Cases } & \multicolumn{2}{|c|}{ 30-Day mortality } & \multirow{2}{*}{$\begin{array}{l}\text { Hospital } \\
\text { mortality }\end{array}$} & \multirow[t]{2}{*}{ VATS } \\
\hline & & Hospital & $\begin{array}{l}\text { After } \\
\text { discharge }\end{array}$ & & \\
\hline $\begin{array}{r}\text { H. Chest } \\
\text { trauma }\end{array}$ & 443 & $20(4.5)$ & 0 & $23(5.2)$ & 289 \\
\hline
\end{tabular}

Table 28 Lung transplantation

\begin{tabular}{|c|c|c|c|c|}
\hline & \multirow[t]{2}{*}{ Cases } & \multicolumn{2}{|c|}{ 30-Day mortality } & \multirow{2}{*}{$\begin{array}{l}\text { Hospital } \\
\text { mortality }\end{array}$} \\
\hline & & Hospital & $\begin{array}{l}\text { After } \\
\text { discharge }\end{array}$ & \\
\hline $\begin{array}{l}\text { Single-lung } \\
\text { transplantation from } \\
\text { brain-dead donor }\end{array}$ & 29 & 0 & 0 & $3(10.3)$ \\
\hline $\begin{array}{l}\text { Bilateral lung } \\
\text { transplantation from } \\
\text { brain-dead donor }\end{array}$ & 28 & 0 & 0 & 0 \\
\hline $\begin{array}{l}\text { Lung transplantation from } \\
\text { living donor }\end{array}$ & 11 & 0 & 0 & 0 \\
\hline Total lung transplantation & 68 & 0 & 0 & $3(4.4)$ \\
\hline $\begin{array}{l}\text { Donor of living donor lung } \\
\text { transplantation }\end{array}$ & 19 & 0 & 0 & 0 \\
\hline
\end{tabular}

Table 29 Video-assisted thoracic surgery

\begin{tabular}{lcccc}
\hline & Cases & \multicolumn{2}{l}{30 -Day mortality } & $\begin{array}{l}\text { Hospital } \\
\text { mortality }\end{array}$ \\
\cline { 3 - 4 } & & Hospital & $\begin{array}{l}\text { After } \\
\text { discharge }\end{array}$ & \\
\hline $\begin{array}{l}\text { Video-assisted } \\
\text { thoracic surgery }\end{array}$ & 68,458 & $\begin{array}{c}218 \\
(0.3)\end{array}$ & $51(0.07)$ & $470(0.7)$ \\
\hline
\end{tabular}

(), mortality \%

(including thoracic sympathectomy 160)\%

Table 27 I. Other respiratory surgery

\begin{tabular}{|c|c|c|c|c|c|}
\hline & \multirow[t]{2}{*}{ Cases } & \multicolumn{2}{|c|}{ 30-Day mortality } & \multirow[t]{2}{*}{ Hospital mortality } & \multirow[t]{2}{*}{ VATS } \\
\hline & & Hospital & After discharge & & \\
\hline I. Other respiratory surgery & 1585 & $38(2.4)$ & $5(0.3)$ & $79(5.0)$ & 1161 \\
\hline Arteriovenous malformation* & 89 & 0 & 0 & 0 & 84 \\
\hline Pulmonary sequestration & 92 & 0 & 0 & 0 & 84 \\
\hline Postoperative bleeding air leakage & 492 & $17(3.5)$ & $1(0.2)$ & $30(6.1)$ & 339 \\
\hline Chylothorax & 62 & $2(3.2)$ & 0 & $4(6.5)$ & 52 \\
\hline Others & 850 & $19(2.2)$ & $4(0.5)$ & $45(5.3)$ & 602 \\
\hline
\end{tabular}

(), mortality \% 
Table 30 Tracheobronchoplasty

\begin{tabular}{|c|c|c|c|c|}
\hline & \multirow[t]{2}{*}{ Cases } & \multicolumn{2}{|c|}{ 30-Day mortality } & \multirow{2}{*}{$\begin{array}{l}\text { Hospital } \\
\text { mortality }\end{array}$} \\
\hline & & Hospital & $\begin{array}{l}\text { After } \\
\text { discharge }\end{array}$ & \\
\hline Tracheobronchoplasty & 774 & $7(0.9)$ & $4(0.5)$ & $16(2.1)$ \\
\hline Trachea & 40 & 0 & 0 & $1(2.5)$ \\
\hline $\begin{array}{l}\text { Sleeve resection with } \\
\text { reconstruction }\end{array}$ & 28 & 0 & 0 & $1(3.6)$ \\
\hline Wedge with simple closure & 4 & 0 & 0 & 0 \\
\hline Wedge with patch closure & 1 & 0 & 0 & 0 \\
\hline $\begin{array}{l}\text { Total laryngectomy with } \\
\text { tracheostomy }\end{array}$ & 0 & 0 & 0 & 0 \\
\hline Others & 7 & 0 & 0 & 0 \\
\hline Carinal reconstruction & 31 & $1(3.2)$ & 0 & $2(6.5)$ \\
\hline Sleeve pneumonectomy & 12 & $2(16.7)$ & 0 & $2(16.7)$ \\
\hline Sleeve lobectomy & 492 & $1(0.2)$ & $2(0.4)$ & $2(0.4)$ \\
\hline Sleeve segmental excision & 14 & 0 & 0 & 0 \\
\hline $\begin{array}{l}\text { Bronchoplasty without lung } \\
\text { resection }\end{array}$ & 29 & $1(3.4)$ & 0 & $1(3.4)$ \\
\hline Others & 156 & $2(1.3)$ & $2(1.3)$ & $8(5.1)$ \\
\hline
\end{tabular}

(), mortality \%

Table 31 Pediatric surgery

\begin{tabular}{lllll}
\hline & Cases & \multicolumn{2}{l}{ 30-Day mortality } & $\begin{array}{l}\text { Hospital } \\
\text { mortality }\end{array}$ \\
\cline { 3 - 5 } & & Hospital & $\begin{array}{l}\text { After } \\
\text { discharge }\end{array}$ & \\
\hline $\begin{array}{l}\text { Pediatric } \\
\text { surgery }\end{array}$ & 292 & $3(1.0)$ & 0 & $3(1.0)$ \\
\hline (), mortality $\%$ & & & &
\end{tabular}

(), mortality \%
Table 32 Combined resection of neighboring organ(s)

\begin{tabular}{|c|c|c|c|c|}
\hline \multirow{4}{*}{$\begin{array}{l}\text { Combined resection of } \\
\text { neighboring organ (s) } \\
\text { Organ resected }\end{array}$} & \multirow[t]{2}{*}{ Cases } & \multicolumn{2}{|c|}{ 30-Day mortality } & \multirow{2}{*}{$\begin{array}{l}\text { Hospital } \\
\text { mortality }\end{array}$} \\
\hline & & Hospital & $\begin{array}{l}\text { After } \\
\text { discharge }\end{array}$ & \\
\hline & 1371 & $7(0.5)$ & 0 & $21(1.5)$ \\
\hline & Cases & \multicolumn{2}{|c|}{ 30-Day mortality } & Hospital \\
\hline & & Hospital & $\begin{array}{l}\text { After } \\
\text { discharge }\end{array}$ & \\
\hline
\end{tabular}

A. Primary lung cancer

$\begin{array}{lrccr}\text { Aorta } & 11 & 0 & 0 & 0 \\ \text { Superior vena cava } & 14 & 1(7.1) & 0 & 1(7.1) \\ \text { Brachiocephalic vein } & 8 & 0 & 0 & 1(12.5) \\ \text { Pericardium } & 108 & 1(0.9) & 0 & 2(1.9) \\ \text { Pulmonary artery } & 127 & 1(0.8) & 0 & 1(0.8) \\ \text { Left atrium } & 19 & 1(5.3) & 0 & 2(10.5) \\ \text { Diaphragm } & 55 & 1(1.8) & 0 & 1(1.8) \\ \text { Chest wall (including } & 352 & 4(1.1) & 0 & 12(3.4) \\ \text { ribs) } & & & & \\ \text { Vertebra } & 19 & 0 & 0 & 0 \\ \text { Esophagus } & 3 & 0 & 0 & 0 \\ \text { Total } & 716 & 9(1.3) & 0 & 20(2.8)\end{array}$

B. Mediastinal tumor

$\begin{array}{lrrlc}\text { Aorta } & 7 & 0 & 0 & 0 \\ \text { Superior vena cava } & 54 & 0 & 0 & 0 \\ \text { Brachiocephalic vein } & 107 & 0 & 0 & 2(1.9) \\ \text { Pericardium } & 351 & 0 & 0 & 3(0.9) \\ \text { Pulmonary artery } & 3 & 0 & 0 & 1(33.3) \\ \text { Left atrium } & 0 & 0 & 0 & 0 \\ \text { Diaphragm } & 21 & 0 & 0 & 0 \\ \text { Chest wall (including } & 10 & 0 & 0 & 0 \\ \text { ribs) } & & & & \\ \text { Vertebra } & 5 & 0 & 0 & 0 \\ \text { Esophagus } & 3 & 0 & 0 & 0 \\ \text { Lung } & 476 & 0 & 0 & 2(0.4) \\ \text { Total } & 1037 & 0 & 0 & 8(0.8)\end{array}$

(), mortality \%

Table 33 Operation of lung cancer invading the chest wall of the apex

\begin{tabular}{|c|c|c|c|c|}
\hline & \multirow[t]{2}{*}{ Cases } & \multicolumn{2}{|c|}{ 30-Day mortality } & \multirow{2}{*}{$\begin{array}{l}\text { Hospital } \\
\text { mortality }\end{array}$} \\
\hline & & Hospital & $\begin{array}{l}\text { After } \\
\text { discharge }\end{array}$ & \\
\hline $\begin{array}{l}\text { 15. Operation of lung } \\
\text { cancer invading the } \\
\text { chest wall of the apex }\end{array}$ & 743 & $7(0.9)$ & $2(0.3)$ & $15(2.0)$ \\
\hline
\end{tabular}


Table 34 Distribution of number of esophageal operations in 2017 in each institution

\begin{tabular}{lccc}
\hline Esophageal surgery & & \\
\hline $\begin{array}{l}\text { Number of } \\
\text { operations in } \\
2017\end{array}$ & $\begin{array}{l}\text { Benign } \\
\text { esophageal } \\
\text { diseases }\end{array}$ & $\begin{array}{l}\text { Malignant } \\
\text { esophageal } \\
\text { disease }\end{array}$ & $\begin{array}{l}\text { Benign }+ \\
\text { malignant }\end{array}$ \\
\hline 0 & 235 & 95 & 76 \\
$1-4$ & 218 & 126 & 114 \\
$5-9$ & 52 & 89 & 96 \\
$10-19$ & 14 & 88 & 98 \\
$20-29$ & 1 & 45 & 47 \\
$30-39$ & 2 & 27 & 26 \\
$40-49$ & 0 & 13 & 21 \\
$\geqq 50$ & 1 & 40 & 45 \\
Total & 523 & 523 & 523 \\
\hline
\end{tabular}

\section{(C) Esophageal Surgery}

During 2017, a total of 12,336 patients with esophageal diseases were registered from 523 institutions (response rate: $92.1 \%$ ) affiliated with the Japanese Association for Thoracic Surgery and/or the Japan Esophageal Society. Among these institutions, there were 139 (26.6\%) where 20 or more patients underwent esophageal surgeries within the year 2017, indicating no definite shift from esophageal procedures to high-volume institutions when compared with the data from 2016 (24.5\%) (Table 34). Among 2427 patients with a benign esophageal disease, 1614 (66.5\%) underwent surgery and $73(3.0 \%)$ underwent endoscopic resection, whereas $740(30.5 \%)$ patients did not undergo surgical treatment (Tables 35, 36). Among 10,554 patients with a malignant esophageal tumor, $8525(80.8 \%)$ underwent resection, esophagectomy was performed for 6319 (74.1\%), and endoscopic mucosal resection (EMR) or endoscopic submucosal dissection (ESD) was performed for 2170 (25.5\%); 2025 (23.8\%) patients did not undergo any resection (Tables 36 and 37). Annual trends among registered inpatients with esophageal diseases have not changed for the past last decades (Fig. 3).

Among benign esophageal diseases (Table 35), hiatal hernia, achalasia, esophageal varices, and esophagitis (including reflux esophagitis) were the most common conditions in Japan. On the other hand, benign esophageal tumors, spontaneous rupture of the esophagus, and congenital esophageal atresia were common diseases that were surgically treated in addition to the aforementioned diseases. Open surgery was performed in 1009 (57.7\%) patients with a benign esophageal disease, with 30-day mortality in $3(0.3 \%)$ patients, whereas thoracoscopic and/ or laparoscopic surgery was performed for 605 (37.5\%) patients, with no instances of 30-day mortality. The difference in these death rates between open and scopic surgery appears to be related to conditions requiring open surgery.

The majority of malignant diseases were carcinomas (Table 36). Among esophageal carcinomas, the incidence of squamous cell carcinoma was $88.8 \%$, whereas that of adenocarcinomas, including Barrett's cancer, was $4.3 \%$. The resection rate for patients with a squamous cell carcinoma was $79.9 \%$, whereas that for patients with adenocarcinoma was $93.8 \%$.

On the basis of location, cancer in the thoracic esophagus was the most common (Table 37). Among 4303 patients (40.9\% of total esophageal malignancies) with superficial esophageal cancers within mucosal and submucosal layers, $6319(60.1 \%)$ patients underwent esophagectomy, whereas 2170 (20.6\%) patients underwent EMR or ESD. The 30-day mortality rate and hospital mortality rate after esophagectomy for patients with a superficial cancer were $0.5 \%$ and $1.0 \%$, respectively.

Multiple primary cancers were observed in 2077 (19.8\%) of all 10,514 patients with esophageal cancer. Synchronous cancer was found in 1008 (9.3\%) patients, whereas metachronous cancer was observed in 1063 $(10.1 \%)$ patients. The stomach and head and neck were common sites for both synchronous and metachronous malignancies (Table 37).

Among esophagectomy procedures, transthoracic esophagectomy via right thoracotomy was most commonly adopted for patients with a superficial cancer, as well as for those with advanced cancer (Table 38). Transhiatal esophagectomy, which is commonly performed in Western countries, was adopted in only $9.1 \%$ of patients with a superficial cancer or advanced cancer who underwent esophagectomy in Japan. Thoracoscopic and/or laparoscopic esophagectomy was adopted for 1434 patients (74.0\%) with a superficial cancer and for 2422 patients (55.3\%) with an advanced cancer. The number of cases of thoracoscopic and/or laparoscopic surgery for superficial or advanced cancer has been increasing for a number of years (Fig. 4).

Combined resection of the neighboring organs during the resection of an esophageal cancer was performed in 296 patients (Tables 38 and 39). Resection of the aorta, together with esophagectomy, was performed in eight cases. Tracheal and/or bronchial resection combined with esophagectomy was performed for 20 patients, with both 30-day mortality rate and hospital mortality rate at $0 \%$. Lung resection combined with esophagectomy was performed for 60 patients, with both 30-day mortality rate and hospital mortality rate at $0 \%$.

Salvage surgery following definitive (chemo)radiotherapy was performed for 230 patients, with 30-day mortality rate at $1.7 \%$ and hospital mortality rate at $2.6 \%$ (Table 38 ). 


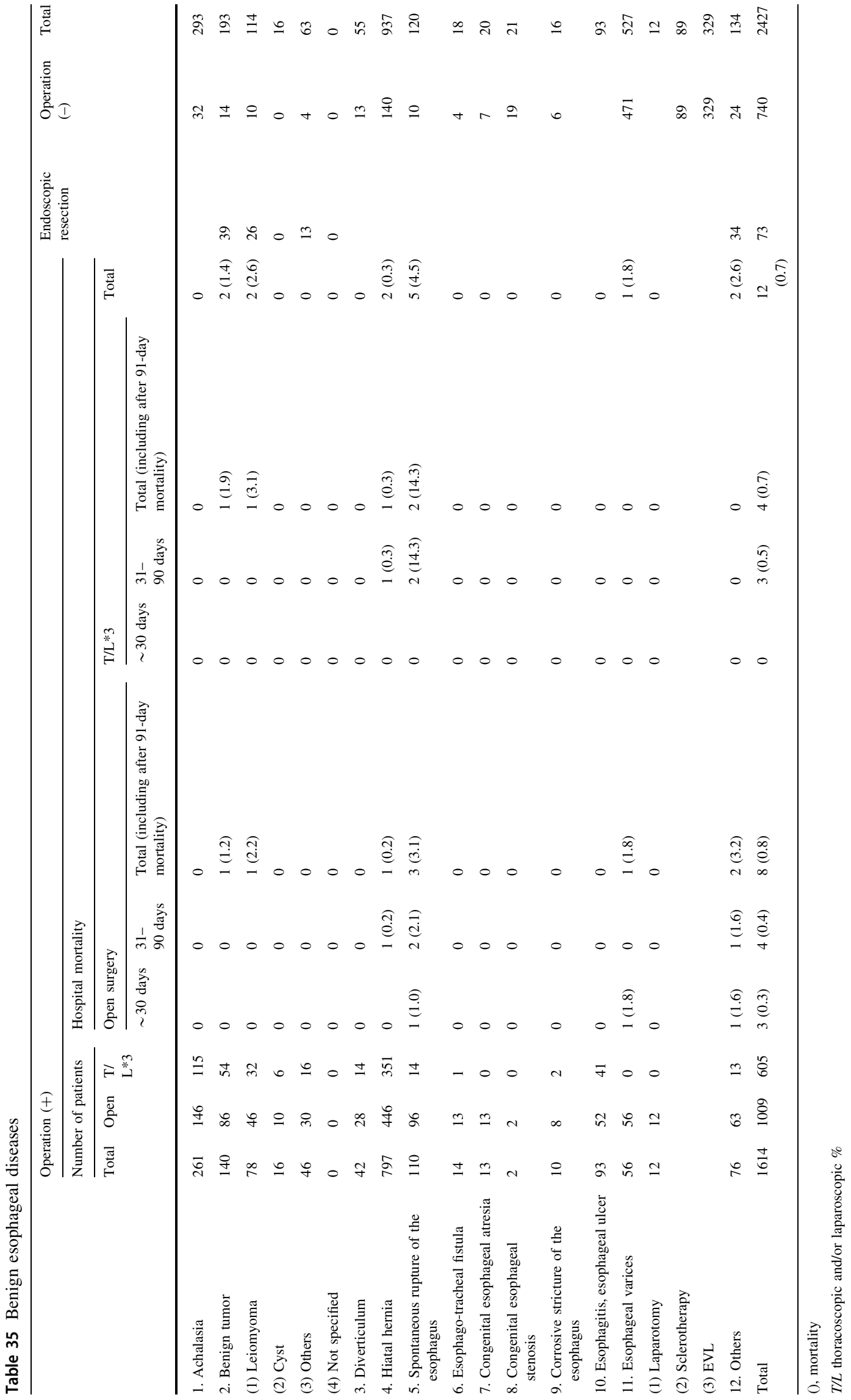


Table 36 Malignant esophageal diseases (histologic classification)

\begin{tabular}{|c|c|c|c|c|}
\hline & & Resection (+) & Resection (-) & Total \\
\hline \multicolumn{2}{|c|}{ Carcinomas } & 8473 & 2025 & 10,498 \\
\hline 1 & Squamous cell carcinoma & 7455 & 1870 & 9325 \\
\hline 2 & Basaloid(-squamous)carcinoma & 86 & 8 & 94 \\
\hline 3 & Carcinosarcoma & 48 & 5 & 53 \\
\hline 4 & Adenocarcinoma in the Barrett's esophagus & 426 & 28 & 454 \\
\hline 5 & Other adenocarcinoma & 369 & 62 & 431 \\
\hline 6 & Adenosquamous carcinoma & 20 & 3 & 23 \\
\hline 7 & Mucoepidermoid carcinoma & 2 & 0 & 2 \\
\hline 8 & Adenoid cystic carcinoma & 4 & 0 & 4 \\
\hline 9 & Endocrine cell carcinoma & 39 & 26 & 65 \\
\hline 10 & Undifferentiated carcinoma & 8 & 6 & 14 \\
\hline 11 & Others & 16 & 17 & 33 \\
\hline \multicolumn{2}{|c|}{ Other malignancies } & 36 & 4 & 40 \\
\hline 1 & Malignant non-epithelial tumors & 8 & 1 & 9 \\
\hline 2 & Malignant melanoma & 20 & 2 & 22 \\
\hline 3 & Other malignant tumors & 8 & 1 & 9 \\
\hline \multicolumn{2}{|c|}{ Not specified } & 16 & 0 & 16 \\
\hline \multicolumn{2}{|l|}{ Total } & 8525 & 2029 & 10,554 \\
\hline
\end{tabular}

Resection: including endoscopic resection 
Table 37 Malignant esophageal disease (clinical characteristics)

\begin{tabular}{|c|c|c|c|c|c|c|c|}
\hline & \multicolumn{4}{|c|}{ Operation $(+)$} & \multirow{3}{*}{$\begin{array}{l}\text { EMR or } \\
\text { ESD }\end{array}$} & \multirow{3}{*}{$\begin{array}{l}\text { Operation } \\
(-)\end{array}$} & \multirow[t]{3}{*}{ Total } \\
\hline & \multirow[t]{2}{*}{ Cases } & \multicolumn{3}{|c|}{ Hospital mortality } & & & \\
\hline & & $\tilde{30}$ days & $\begin{array}{l}31- \\
90 \text { days }\end{array}$ & $\begin{array}{l}\text { Total (including after 91-day } \\
\text { mortality) }\end{array}$ & & & \\
\hline 1. Esophageal cancer & 6319 & $33(0.5)$ & $30(0.5)$ & $83(1.3)$ & 2170 & 2025 & 10,514 \\
\hline \multicolumn{8}{|l|}{ Location } \\
\hline (1) Cervical esophagus & 223 & 0 & 0 & $2(0.9)$ & 102 & 142 & 467 \\
\hline (2) Thoracic esophagus & 5117 & $30(0.6)$ & $27(0.5)$ & $75(1.5)$ & 1637 & 1682 & 8436 \\
\hline (3) Abdominal esophagus & 718 & $2(0.3)$ & $3(0.4)$ & $5(0.7)$ & 119 & 99 & 936 \\
\hline (4) Multiple cancers & 261 & $1(0.4)$ & 0 & $1(0.4)$ & 216 & 41 & 518 \\
\hline (5) Others/not described & 0 & 0 & 0 & 0 & 96 & 61 & 157 \\
\hline \multicolumn{8}{|l|}{ Tumor depth } \\
\hline (A) Superficial cancer (T1) & 1938 & $10(0.5)$ & $6(0.3)$ & $19(1.0)$ & 2162 & 203 & 4303 \\
\hline Mucosal cancer $($ T1a) & 346 & $1(0.3)$ & $1(0.3)$ & $4(1.2)$ & 1557 & 39 & 1942 \\
\hline $\begin{array}{l}\text { (B) Advanced cancer (T2- } \\
\text { T4) }\end{array}$ & 4377 & $23(0.5)$ & $24(0.5)$ & $64(1.5)$ & 5 & 1766 & 6148 \\
\hline (C) Not specified & 4 & 0 & 0 & 0 & 3 & 56 & 63 \\
\hline 2. Multiple primary cancers & 1167 & $7(0.6)$ & $9(0.8)$ & $19(1.6)$ & 611 & 299 & 2077 \\
\hline 1) Synchronous & 658 & $6(0.9)$ & $8(1.2)$ & $16(2.4)$ & 260 & 145 & 1063 \\
\hline (1) Head and neck & 231 & $1(0.4)$ & $2(0.9)$ & $4(1.7)$ & 133 & 50 & 414 \\
\hline (2) Stomach & 203 & $3(1.5)$ & $4(2.0)$ & $9(4.4)$ & 66 & 34 & 303 \\
\hline (3) Colorectum & 83 & $1(1.2)$ & 0 & $1(1.2)$ & 16 & 12 & 111 \\
\hline (4) Lung & 42 & $1(2.4)$ & 0 & $1(2.4)$ & 8 & 17 & 67 \\
\hline (5) Pancreas & 2 & 0 & 0 & 0 & 3 & 2 & 7 \\
\hline (6) Liver & 6 & 0 & 0 & 0 & 1 & 2 & 9 \\
\hline (7) Others & 56 & 0 & $1(1.8)$ & 0 & 16 & 17 & 89 \\
\hline (8) Triple cancers & 35 & 0 & $1(2.9)$ & $1(2.9)$ & 17 & 11 & 63 \\
\hline (9) Unknown & 0 & 0 & 0 & 0 & 0 & 0 & 0 \\
\hline 2) Metachronous & 509 & $1(0.2)$ & $1(0.2)$ & $3(0.6)$ & 351 & 154 & 1014 \\
\hline (1) Head and neck & 98 & 0 & 0 & 0 & 117 & 31 & 246 \\
\hline (2) Stomach & 104 & 0 & 0 & 0 & 76 & 31 & 211 \\
\hline (3) Colorectum & 70 & 0 & 0 & $1(1.4)$ & 35 & 20 & 125 \\
\hline (4) Lung & 47 & 0 & 0 & 0 & 10 & 19 & 76 \\
\hline (5) Pancreas & 3 & 0 & 0 & 0 & 2 & 0 & 5 \\
\hline (6) Liver & 5 & 0 & 0 & 0 & 1 & 2 & 8 \\
\hline (7) Others & 148 & $1(0.7)$ & $1(0.7)$ & $2(1.4)$ & 60 & 31 & 239 \\
\hline (8) Triple cancers & 34 & 0 & 0 & 0 & 49 & 20 & 103 \\
\hline (9) Unknown & 0 & 0 & 0 & 0 & 1 & 0 & 1 \\
\hline Unknown & 0 & 0 & 0 & 0 & 0 & 0 & 0 \\
\hline
\end{tabular}

(), mortality \%

$E M R$ endoscopic mucosal resection (including endoscopic submucosal dissection) 


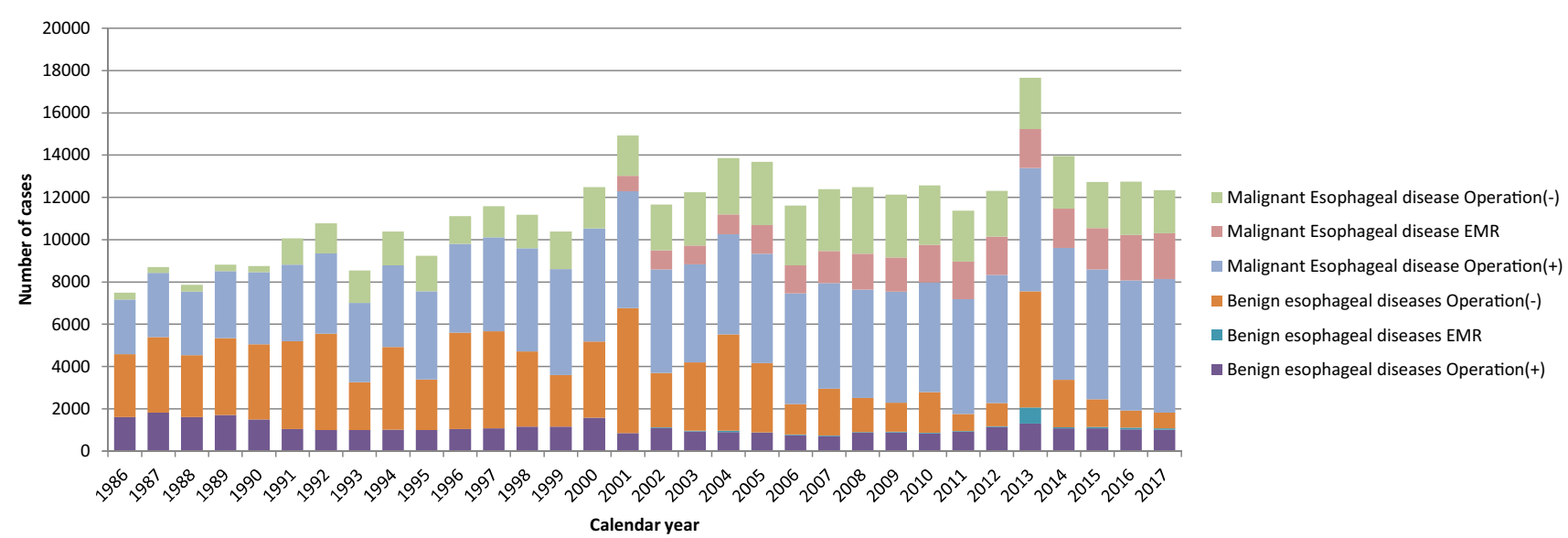

Fig. 3 Annual trend of inpatients with esophageal diseases. EMR endoscopic mucosal resection (including endoscopic submucosal) 
Table 38 Malignant esophageal disease (surgical procedures)

\begin{tabular}{|c|c|c|c|c|c|c|c|c|c|}
\hline & \multicolumn{8}{|c|}{ Operation $(+)$} & \multirow{4}{*}{$\begin{array}{l}\text { EMR or } \\
\text { ESD }\end{array}$} \\
\hline & \multirow{3}{*}{ Cases } & \multirow{2}{*}{\multicolumn{3}{|c|}{ Hospital mortality }} & \multicolumn{4}{|c|}{ Thoracoscopic and/or laparoscopic procedure } & \\
\hline & & & & & \multirow[t]{2}{*}{ Cases } & \multicolumn{3}{|c|}{ Hospital mortality } & \\
\hline & & $\widetilde{30}$ days & $\begin{array}{l}31- \\
90 \text { days }\end{array}$ & $\begin{array}{l}\text { Total (including after } \\
\text { 91-day mortality) }\end{array}$ & & $\widetilde{30}$ days & $\begin{array}{l}31- \\
90 \text { days }\end{array}$ & $\begin{array}{l}\text { Total (including after } 91- \\
\text { day mortality) }\end{array}$ & \\
\hline Superficial cancer (T1) & 1938 & $10(0.5)$ & $6(0.3)$ & $19(1.0)$ & 1434 & $8(0.6)$ & $5(0.3)$ & $15(1.0)$ & 2162 \\
\hline Mucosal cancer $(T l a)$ & 346 & $1(0.3)$ & $1(0.3)$ & $4(1.2)$ & 210 & $1(0.5)$ & $1(0.5)$ & $4(1.9)$ & 1557 \\
\hline Esophagectomy & 1938 & $10(0.5)$ & $6(0.3)$ & $19(1.0)$ & 1434 & $8(0.6)$ & $5(0.3)$ & $15(1.0)$ & 2162 \\
\hline $\begin{array}{l}\text { (1) Transhiatal esophagectomy, } \\
\text { mediascope-assisted esophagectomy }\end{array}$ & 176 & 0 & 0 & $1(0.6)$ & 85 & 0 & 0 & $1(1.2)$ & \\
\hline $\begin{array}{l}\text { (2) Transthoracic (rt.) esophagectomy and } \\
\text { reconstruction }\end{array}$ & 1649 & $10(0.6)$ & $5(0.3)$ & $16(1.0)$ & 1296 & $8(0.6)$ & $5(0.4)$ & $13(1.0)$ & \\
\hline $\begin{array}{l}\text { (3) Transthoracic (lt.) esophagectomy and } \\
\text { reconstruction }\end{array}$ & 31 & 0 & $1(3.2)$ & $1(3.2)$ & 9 & 0 & 0 & 0 & \\
\hline $\begin{array}{l}\text { (4) Cervical esophageal resection and } \\
\text { reconstruction }\end{array}$ & 20 & 0 & 0 & 0 & 5 & 0 & 0 & 0 & \\
\hline (5) Robot-assisted esophagectomy & 19 & 0 & 0 & 0 & 19 & 0 & 0 & 0 & \\
\hline (6) Others & 28 & 0 & 0 & 0 & 12 & 0 & 0 & 0 & \\
\hline (7) Esophagectomy without reconstruction & 9 & 0 & 0 & $1(11.1)$ & 2 & 0 & 0 & $1(50.0)$ & \\
\hline (8) Not specified & 6 & 0 & 0 & 0 & 6 & 0 & 0 & 0 & \\
\hline \multicolumn{10}{|l|}{ Advanced cancer (T2-T4) } \\
\hline Esophagectomy & 4377 & $23(0.5)$ & $24(0.5)$ & $64(1.5)$ & 2422 & $11(0.5)$ & $10(0.4)$ & $28(1.2)$ & 5 \\
\hline $\begin{array}{l}\text { (1) Transhiatal esophagectomy, } \\
\text { mediascope-assisted esophagectomy }\end{array}$ & 240 & $1(0.4)$ & $1(0.4)$ & $2(0.8)$ & 92 & 0 & $1(1.1)$ & $1(1.1)$ & \\
\hline $\begin{array}{l}\text { (2) Transthoracic (rt.) esophagectomy and } \\
\text { reconstruction }\end{array}$ & 3818 & $21(0.6)$ & $20(0.5)$ & $55(1.4)$ & 2255 & $10(0.4)$ & $9(0.4)$ & $25(1.1)$ & \\
\hline $\begin{array}{l}\text { (3) Transthoracic (lt.) esophagectomy and } \\
\text { reconstruction }\end{array}$ & 89 & 0 & 0 & 0 & 4 & 0 & 0 & 0 & \\
\hline $\begin{array}{l}\text { (4) Cervical esophageal resection and } \\
\text { reconstruction }\end{array}$ & 105 & 0 & 0 & $1(1.0)$ & 6 & 0 & 0 & 0 & \\
\hline (5) Robot-assisted esophagectomy & 19 & 0 & 0 & 0 & 19 & 0 & 0 & 0 & \\
\hline (6) Others & 57 & $1(1.8)$ & $1(1.8)$ & $2(3.5)$ & 22 & $1(4.5)$ & 0 & $1(4.5)$ & \\
\hline (7) Esophagectomy without reconstruction & 43 & 0 & $2(4.7)$ & $4(9.3)$ & 19 & 0 & 0 & $1(5.3)$ & \\
\hline (8) Not specified & 6 & 0 & 0 & 0 & 5 & 0 & 0 & 0 & \\
\hline (Depth not specified) & 4 & 0 & 0 & 0 & 3 & 0 & 0 & 0 & 3 \\
\hline Combined resection of other organs & 296 & $3(1.0)$ & $3(1.0)$ & $7(2.4)$ & & & & & \\
\hline (1) Aorta & 8 & $1(12.5)$ & 0 & $1(12.5)$ & & & & & \\
\hline (2) Trachea, bronchus & 20 & 0 & 0 & $1(5.0)$ & & & & & \\
\hline (3) Lung & 60 & 0 & 0 & 0 & & & & & \\
\hline (4) Others & 208 & $2(1.0)$ & $3(1.4)$ & $5(2.4)$ & & & & & \\
\hline Unknown & 0 & 0 & 0 & 0 & & & & & \\
\hline Salvage surgery & 230 & $4(1.7)$ & $1(0.4)$ & $6(2.6)$ & 70 & $1(1.4)$ & 0 & $1(1.4)$ & 25 \\
\hline
\end{tabular}




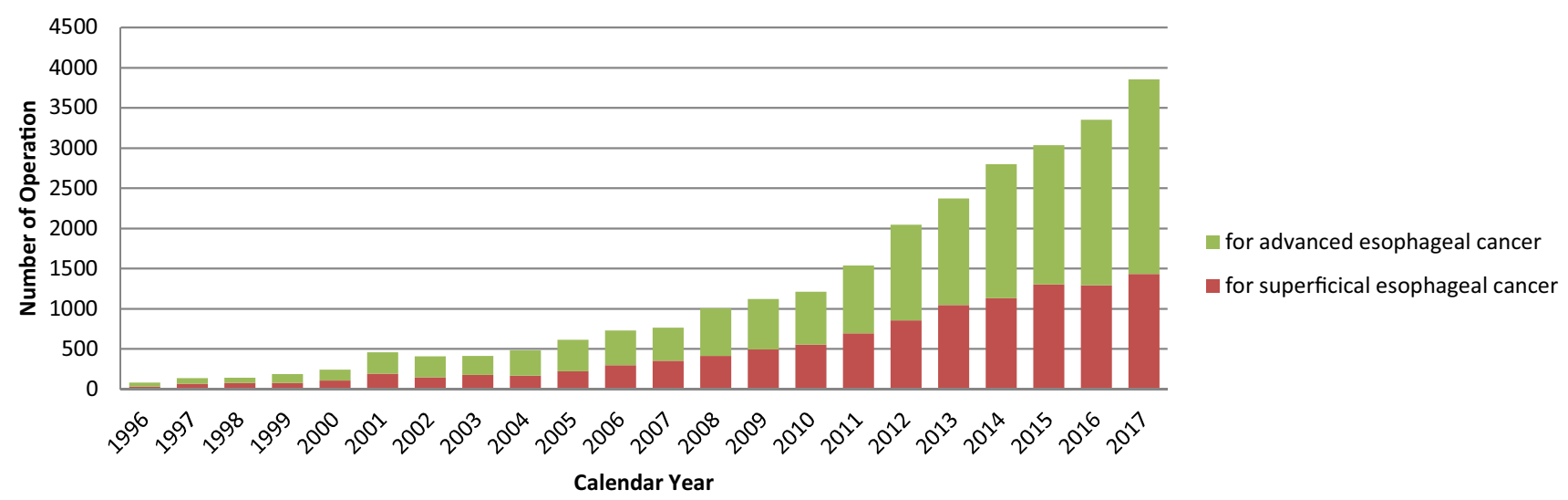

Fig. 4 Annual trend of video-assisted esophagectomy for esophageal malignancy

Table 39 Mortality after combined resection of the neighbouring organs

\begin{tabular}{|c|c|c|c|c|c|c|c|c|c|c|c|c|c|c|c|}
\hline \multirow[t]{3}{*}{ Year } & \multirow{2}{*}{\multicolumn{3}{|c|}{ Esophagectomy }} & \multicolumn{12}{|c|}{ Combined resection } \\
\hline & & & & \multicolumn{3}{|c|}{ Aorta } & \multicolumn{3}{|c|}{ Tracheobronchus } & \multicolumn{3}{|l|}{ Lung } & \multicolumn{3}{|c|}{ Others } \\
\hline & a & b & $\mathrm{c}$ & $\mathrm{a}$ & $\mathrm{b}$ & $\mathrm{c}$ & $\mathrm{a}$ & $\mathrm{b}$ & $\mathrm{c}$ & $\mathrm{a}$ & $\mathrm{b}$ & $\mathrm{c}$ & $\mathrm{a}$ & $\mathrm{b}$ & $\mathrm{c}$ \\
\hline 1996 & 4194 & 120 & $2.86 \%$ & 7 & 3 & $42.86 \%$ & 24 & 0 & $0.00 \%$ & 50 & 2 & $4.00 \%$ & 78 & 4 & $5.13 \%$ \\
\hline 1997 & 4441 & 127 & $2.86 \%$ & 1 & 0 & $0.00 \%$ & 34 & 5 & $14.71 \%$ & 56 & 1 & $1.79 \%$ & 94 & 3 & $3.19 \%$ \\
\hline 1998 & 4878 & 136 & $2.79 \%$ & 4 & 0 & $0.00 \%$ & 29 & 0 & $0.00 \%$ & 74 & 1 & $1.35 \%$ & 128 & 2 & $1.56 \%$ \\
\hline 1999 & 5015 & 116 & $2.31 \%$ & 5 & 0 & $0.00 \%$ & 23 & 2 & $8.70 \%$ & 68 & 0 & $0.00 \%$ & 122 & 1 & $0.82 \%$ \\
\hline 2000 & 5350 & 81 & $1.51 \%$ & 2 & 0 & $0.00 \%$ & 23 & 2 & $8.70 \%$ & 69 & 0 & $0.00 \%$ & 96 & 1 & $1.04 \%$ \\
\hline 2001 & 5521 & 110 & $1.99 \%$ & 1 & 0 & $0.00 \%$ & 26 & 1 & $3.85 \%$ & 83 & 3 & $3.61 \%$ & 99 & 2 & $2.02 \%$ \\
\hline 2002 & 4904 & 66 & $1.35 \%$ & 3 & 1 & $33.33 \%$ & 20 & 2 & $10.00 \%$ & 63 & 0 & $0.00 \%$ & 63 & 1 & $1.59 \%$ \\
\hline 2003 & 4639 & 45 & $0.97 \%$ & 0 & 0 & $0.00 \%$ & 24 & 2 & $8.33 \%$ & 58 & 0 & $0.00 \%$ & 88 & 1 & $1.14 \%$ \\
\hline 2004 & 4739 & 64 & $1.35 \%$ & 2 & 0 & $0.00 \%$ & 17 & 0 & $0.00 \%$ & 59 & 5 & $8.47 \%$ & 119 & 2 & $1.68 \%$ \\
\hline 2005 & 5163 & 52 & $1.01 \%$ & 1 & 0 & $0.00 \%$ & 11 & 1 & $9.09 \%$ & 67 & 1 & $1.49 \%$ & 73 & 1 & $1.37 \%$ \\
\hline 2006 & 5236 & 63 & $1.20 \%$ & 0 & 0 & $0.00 \%$ & 17 & 0 & $0.00 \%$ & 62 & 2 & $3.23 \%$ & 122 & 3 & $2.46 \%$ \\
\hline 2007 & 4990 & 60 & $1.20 \%$ & 0 & 0 & $0.00 \%$ & 25 & 1 & $4.00 \%$ & 44 & 1 & $2.27 \%$ & 138 & 2 & $1.45 \%$ \\
\hline 2008 & 5124 & 63 & $1.23 \%$ & 0 & 0 & $0.00 \%$ & 17 & 1 & $5.88 \%$ & 48 & 1 & $2.08 \%$ & 185 & 0 & $0.00 \%$ \\
\hline 2009 & 5260 & 63 & $1.20 \%$ & 0 & 0 & $0.00 \%$ & 19 & 2 & $10.53 \%$ & 58 & 2 & $3.45 \%$ & 211 & 3 & $1.42 \%$ \\
\hline 2010 & 5180 & 45 & $0.87 \%$ & 2 & 0 & $0.00 \%$ & 33 & 0 & $0.00 \%$ & 58 & 0 & $0.00 \%$ & 245 & 5 & $2.04 \%$ \\
\hline 2011 & 5430 & 38 & $0.70 \%$ & 4 & 0 & $0.00 \%$ & 26 & 0 & $0.00 \%$ & 41 & 0 & $0.00 \%$ & 179 & 5 & $2.79 \%$ \\
\hline 2012 & 6055 & 47 & $0.78 \%$ & 2 & 0 & $0.00 \%$ & 23 & 1 & $4.35 \%$ & 69 & 0 & $0.00 \%$ & 240 & 1 & $0.42 \%$ \\
\hline 2013 & 5824 & 41 & $0.70 \%$ & 2 & 0 & $0.00 \%$ & 44 & 0 & $0.00 \%$ & 77 & 1 & $1.30 \%$ & 156 & 3 & $1.92 \%$ \\
\hline 2014 & 6244 & 47 & $0.75 \%$ & 2 & 0 & $0.00 \%$ & 24 & 0 & $0.00 \%$ & 77 & 3 & $3.90 \%$ & 227 & 3 & $1.32 \%$ \\
\hline 2015 & 6151 & 39 & $0.63 \%$ & 3 & 0 & $0.00 \%$ & 15 & 0 & $0.00 \%$ & 67 & 3 & $4.48 \%$ & 266 & 4 & $1.50 \%$ \\
\hline 2016 & 6158 & 40 & $0.65 \%$ & 3 & 0 & $0.00 \%$ & 12 & 0 & $0.00 \%$ & 56 & 0 & $0.00 \%$ & 155 & 1 & $0.65 \%$ \\
\hline 2017 & 6319 & 33 & $0.52 \%$ & 8 & 1 & $12.50 \%$ & 20 & 0 & $0.00 \%$ & 60 & 0 & $0.00 \%$ & 208 & 2 & $0.96 \%$ \\
\hline Total & 116,815 & 1496 & $1.28 \%$ & 52 & 5 & $9.62 \%$ & 506 & 20 & $3.95 \%$ & 1364 & 26 & $1.91 \%$ & 3292 & 50 & $1.52 \%$ \\
\hline
\end{tabular}

a: number of patients who underwent the operation

b: number of patients died within 30 days after operation

c: \% ratio of b/a, i.e., direct operative mortality 
We aim to continue our efforts to gather all-encompassing survey data via more active collaboration with the Japan Esophageal Society and other related institutions.

Acknowledgements On behalf of the Japanese Association for Thoracic Surgery, the authors thank the Heads of the Affiliate and Satellite Institutes of Thoracic Surgery for their cooperation, and the Councilors of the Japan Esophageal Society.

Open Access This article is licensed under a Creative Commons Attribution 4.0 International License, which permits use, sharing, adaptation, distribution and reproduction in any medium or format, as long as you give appropriate credit to the original author(s) and the source, provide a link to the Creative Commons licence, and indicate if changes were made. The images or other third party material in this article are included in the article's Creative Commons licence, unless indicated otherwise in a credit line to the material. If material is not included in the article's Creative Commons licence and your intended use is not permitted by statutory regulation or exceeds the permitted use, you will need to obtain permission directly from the copyright holder. To view a copy of this licence, visit http://creativecommons. org/licenses/by/4.0/.

\section{References}

1. Shimizu H, Endo S, Natsugoe S, et al. Thoracic and cardiovascular surgery in Japan during 2016 - annual report by the Japanese Association for Thoracic Surgery. Gen Thorac Cardiovasc Surg. 2019;67:377-411.

2. Ueda Y, Fujii Y, Kuwano H. Thoracic and cardiovascular surgery in Japan during 2007-annual report by the Japanese Association for Thoracic Surgery. Gen Thorac Cardiovasc Surg. 2009;57:488513.

3. Masuda M, Kuwano H, Okumura M, et al. Thoracic and cardiovascular surgery in Japan during 2012 - annual report by the Japanese Association for Thoracic Surgery. Gen Thorac Cardiovasc Surg. 2014;62:734-64.

4. Kazui T, Wada H, Fujita $H$. Thoracic and cardiovascular surgery in Japan during 2003 - annual report by the Japanese Association for Thoracic Surgery. Jpn J Thorac Cardiovasc Surg. 2005;53:517-36.

5. Kazui T, Osada H, Fujita H. Thoracic and cardiovascular surgery in Japan during 2004-annual report by the Japanese Association for Thoracic Surgery. Jpn J Thorac Cardiovasc Surg. 2006;54:363-86.

Publisher's Note Springer Nature remains neutral with regard to jurisdictional claims in published maps and institutional affiliations.

\section{Affiliations}

Committee for Scientific Affairs, The Japanese Association for Thoracic Surgery ${ }^{1} \cdot$ Hideyuki Shimizu $^{2}$. Morihito Okada ${ }^{3}$ - Akira Tangoku ${ }^{4}$ - Yuichiro Doki ${ }^{5}$ Shunsuke Endo ${ }^{6}$ - Hirotsugu Fukuda ${ }^{7}$.

Yasutaka Hirata $^{8}$ - Hisashi Iwata ${ }^{9}$. Junjiro Kobayashi ${ }^{10}$. Hiraku Kumamaru ${ }^{11}$. Hiroaki Miyata ${ }^{12}$. Noboru Motomura $^{13}$. Shoji Natsugoe ${ }^{14}$. Soji Ozawa ${ }^{15} \cdot$ Yoshikatsu Saiki $^{16}$ - Aya Saito $^{13} \cdot$ Hisashi Saji $^{17}$. Yukio Sato ${ }^{18} \cdot$ Tsuyoshi Taketani $^{19} \cdot$ Kazuo Tanemoto $^{20} \cdot$ Wataru Tatsuishi $^{21} \cdot$ Yasushi Toh $^{22}$. Hiroyuki Tsukihara $^{8} \cdot$ Masayuki Watanabe $^{23} \cdot$ Hiroyuki Yamamoto $^{12} \cdot$ Kohei Yokoi $^{24} \cdot$ Yutaka Okita $^{25}$

Hideyuki Shimizu

survey-adm@umin.net

1 Committee for Scientific Affairs, The Japanese Association for Thoracic Surgery, Tokyo, Japan

2 Department of Cardiovascular Surgery, Keio University, 35, Shinanomachi, Shinjuku-Ku, Tokyo, Japan

3 Department of Surgical Oncology, Hiroshima University, Hiroshima, Japan

4 Department of Thoracic, Endocrine Surgery and Oncology, Institute of BioMedicine, Tokushima University Graduate School, Tokushima, Japan

5 Department of Gastroenterological Surgery, Osaka University, Suita, Osaka, Japan

6 Department of Thoracic Surgery, Jichi Medical University, Shimotsuke, Japan

7 Department of Cardiac and Vascular Surgery, Dokkyo Medical University School of Medicine, Shimotsuga-gun, Tochigi, Japan

8 Department of Cardiac Surgery, The University of Tokyo Hospital, Tokyo, Japan

9 Department of General Thoracic Surgery, Gifu University Hospital, Gifu, Japan
10 Department of Cardiovascular Surgery, National Cerebral and Cardiovascular Center, Suita, Japan

11 Department of Healthcare Quality Assessment, Graduate School of Medicine, University of Tokyo, Tokyo, Japan

12 Department of Health Policy and Management, Keio University, Tokyo, Japan

13 Department of Cardiovascular Surgery, Toho University Sakura Medical Center, Sakura, Japan

14 Department of Digestive Surgery, Breast and Thyroid Surgery, Kagoshima University Graduate School of Medicine, Kagoshima, Japan

15 Department of Gastroenterological Surgery, Tokai University School of Medicine, Isehara, Japan

16 Division of Cardiovascular Surgery, Tohoku University Graduate School of Medicine, Sendai, Japan

17 Department of Chest Surgery, St. Marianna University School of Medicine, Kawasaki, Japan

18 Department of Thoracic Surgery, University of Tsukuba, Tsukuba, Japan

19 Department of Cardiovascular Surgery, Mitsui Memorial Hospital, Tokyo, Japan 
20 Department of Cardiovascular Surgery, Kawasaki Medical School, Kurashiki, Japan

21 Division of Cardiovascular Surgery, Department of General Surgical Science, Gunma University, Maebashi, Japan

22 Department of Gastroenterological Surgery, National Hospital Organization Kyushu Cancer Center, Fukuoka, Japan
23 Department of Gastroenterological Surgery, Cancer Institute Hospital of Japanese Foundation for Cancer Research, Tokyo, Japan

24 Chunichi Hospital, Nagoya, Japan

25 Cardio-Aortic Center, Takatsuki General Hospital, Takatsuki, Japan 